\title{
Liability Games
}

Citation for published version (APA):

Csoka, P., \& Herings, P. J-J. (2017). Liability Games. Maastricht University, Graduate School of Business and Economics. GSBE Research Memoranda No. 031 https://doi.org/10.26481/umagsb.2017031

\section{Document status and date:}

Published: 07/12/2017

DOI:

10.26481/umagsb.2017031

Document Version:

Publisher's PDF, also known as Version of record

\section{Please check the document version of this publication:}

- A submitted manuscript is the version of the article upon submission and before peer-review. There can be important differences between the submitted version and the official published version of record.

People interested in the research are advised to contact the author for the final version of the publication, or visit the DOI to the publisher's website.

- The final author version and the galley proof are versions of the publication after peer review.

- The final published version features the final layout of the paper including the volume, issue and page numbers.

Link to publication

\footnotetext{
General rights rights.

- You may freely distribute the URL identifying the publication in the public portal. please follow below link for the End User Agreement:

www.umlib.nl/taverne-license

Take down policy

If you believe that this document breaches copyright please contact us at:

repository@maastrichtuniversity.nl

providing details and we will investigate your claim.
}

Copyright and moral rights for the publications made accessible in the public portal are retained by the authors and/or other copyright owners and it is a condition of accessing publications that users recognise and abide by the legal requirements associated with these

- Users may download and print one copy of any publication from the public portal for the purpose of private study or research.

- You may not further distribute the material or use it for any profit-making activity or commercial gain

If the publication is distributed under the terms of Article $25 \mathrm{fa}$ of the Dutch Copyright Act, indicated by the "Taverne" license above, 


\section{Maastricht University}

Péter Csóka,

P. Jean-Jacques Herings

\section{Liability Games}

$\mathrm{RM} / 17 / 031$

\section{GSBE}

Maastricht University School of Business and Economics

Graduate School of Business and Economics

P.O Box 616

NL- 6200 MD Maastricht

The Netherlands 


\title{
Liability Games*
}

\section{Péter Csóka ${ }^{\dagger} \quad$ P. Jean-Jacques Herings ${ }^{\ddagger}$}

November 29, 2017

\begin{abstract}
A firm has liabilities towards a group of creditors. We analyze the question of how to distribute the asset value of the firm among the creditors and the firm itself. Compared to standard bankruptcy games as studied in the game theory literature, we introduce the firm as an explicit player and define a new class of transferable utility games called liability games. Liability games are superadditive, constant sum, partially convex, and partially concave. The core of a liability game is empty if and only if the firm is insolvent and has multiple positive liabilities.

We analyze the nucleolus of the game and show that allocating the asset value of the firm using the nucleolus satisfies efficiency, non-negativity, and liabilities boundedness. We prove that at the nucleolus, the firm gets a strictly higher amount than its stand-alone value if and only if the firm is insolvent and has multiple positive liabilities. The firm is using the threat to pay others to get debt forgiveness and is able to keep a positive amount of its assets. We provide conditions under which the nucleolus coincides with a generalized truncated proportional rule, assigning a non-negative payment to the firm and distributing the remainder in proportion to the liabilities, truncated by the asset value of the firm.
\end{abstract}

Keywords: Insolvency, debt forgiveness, bankruptcy games, nucleolus, proportional rule.

JEL Classification: C71, G10

${ }^{*}$ We would like to thank Sjur Didrik Flåm, Miklós Pintér, Tamás Solymosi, Balázs Sziklai and participants of SING13 and the 8th Annual Financial Market Liquidity Conference for helpful comments.

†Department of Finance, Corvinus Business School, Corvinus University of Budapest and "Momentum" Game Theory Research Group, Centre for Economic and Regional Studies, Hungarian Academy of Sciences. E-mail: peter.csoka@uni-corvinus.hu. Péter Csóka thanks funding from National Research, Development and Innovation Office - NKFIH, K-120035.

${ }_{\ddagger}^{\ddagger}$ Department of Economics, Maastricht University, P.O. Box 616, 6200 MD, Maastricht, The Netherlands. E-mail: P.Herings@maastrichtuniversity.nl. 


\section{Introduction}

It is often the case that insolvent agents agree with their creditors to decrease the value of their liabilities by reducing the principal, restructuring the payments, or getting longer maturities. For each creditor, the difference between the value of the old liability and the legally binding, but lower level of new liability is called the debt forgiveness of the creditor. For sovereign defaults, about 30-40 \% debt forgiveness is documented by Arslanalp and Henry (2005), D'Erasmo (2008), and Benjamin and Wright (2009).

Insolvent agents can be either countries, states, firms, individuals, or other organizations. Throughout the paper, we will stick to the term firm. A liability problem consists of a firm with a certain asset value and liabilities towards a group of creditors. The question is how to distribute the asset value of the firm among the creditors and the firm itself. Given a liability problem, a liability rule assigns a vector of payments to the creditors and to the firm. Payment vectors should satisfy non-negativity as the firm has limited liability and no creditor should be asked to pay, liabilities boundedness as the firm does not pay in excess of its liabilities, and efficiency meaning that the sum of the payments should be equal to the firm's asset value. A frequently studied liability rule is the proportional rule, which assigns a payment of zero to the firm and distributes the asset value in proportion to the liabilities to the firm's creditors. We introduce generalized proportional rules as those which allows for a non-negative payment to the firm and a proportional distribution of the remainder.

Sturzenegger and Zettelmeyer (2007) and Chatterjee and Eyigungor (2015) note that there is no settled theory for the renegotiations concerning the distribution of the asset value of the firm, but creditors may join ad hoc committees and try to force the firm to pay them first. We make use of the theory of transferable utility games and can thereby omit the details of the renegotiation process. The resulting transferable utility games are called liability games.

Compared to standard bankruptcy games as studied in the game theoretical literature, see O'Neill (1982) for a seminal contribution and Thomson (2003), Thomson (2013), and Thomson (2015) for recent surveys, we introduce the firm as an explicit player. The worth of a coalition in a liability game has the usual interpretation. It is what the members of a coalition can guarantee for themselves, irrespective of what outsiders do. More specifically, given a coalition and its complement, the firm first makes payments to the coalition it belongs to, up to the value of the liabilities in the firm's coalition, and then pays to the complementary coalition. If the firm is solvent, its coalition can also keep what is left from the asset value after paying all creditors.

Liability games turn out to have some special properties. They feature both concavity and convexity: the subgame restricted to all creditors is convex, whereas the game obtained 
by restricting attention to the coalitions containing the firm is concave. Liability games are superadditive: there is no loss of merging disjoint coalitions. Moreover, in a liability game, the worth of a coalition plus the worth of the complementary coalition is always equal to the asset value, that is, a liability game is a constant sum game (von Neumann and Morgenstern, 1944).

The core of a liability game consists of those payment vectors where no coalition of the players can complain, that is, where every coalition of players receives at least its worth. We show that the core of a liability game is non-empty if and only if the game is additive, that is, the worth of a coalition is simply the sum of the worths of its members, so there is no synergy from forming a coalition. We also show that a liability game is additive if and only if the firm is solvent or it is insolvent and has only one positive liability.

Since the core of a liability game is empty when the firm is insolvent and has multiple positive liabilities, a solution concept minimizing the complaints of the coalitions is a natural candidate for a liability rule, which brings us to the nucleolus (Schmeidler, 1969). We show that the nucleolus satisfies the properties required for a liability rule: efficiency, non-negativity, and liabilities boundedness, that is, the payment to a creditor can be at most as much as his claim. We show that the nucleolus coincides with the Shapley value (Shapley, 1953) for the case where the firm is solvent, or insolvent with at most two positive liabilities.

We also prove that at the nucleolus, the firm gets a strictly higher amount than its stand-alone value if and only if the firm is insolvent and has multiple positive liabilities. Thus the firm needs the threat to pay others to end up with a positive asset value and obtain some debt forgiveness. To provide an upper bound on how much an insolvent firm with multiple positive liabilities can gain at the nucleolus as a liability rule, we show that it ends up with at most half of the asset value. We show that in this case, creditors with higher claims get higher payments, but they also have higher debt forgiveness. In fact, there is a threshold of liabilities, below which there is no debt forgiveness, above which there is positive debt forgiveness. Thus creditors with smaller claims may not be threatened. Finally, we provide conditions under which the nucleolus coincides with a generalized truncated proportional rule. This generalized truncated proportional rule assigns a nonnegative payment to the firm and distributes the remainder in proportion to the liabilities that result after truncation by the asset value of the firm. We present a closed-form expression for the non-negative payment to the firm, thereby pinning down the generalized truncated proportional rule that coincides with the nucleolus.

There is a large game-theoretic literature on problems where the bankrupt agent is not treated as a player, but as an exogenously given estate. We refer to such problems as bankruptcy problems and to the induced transferable utility games as bankruptcy games. 
In bankruptcy problems, it is standard to allocate the entire estate to the claimants. For surveys of this literature, we refer the reader to Thomson (2003), Thomson (2013), and Thomson (2015).

Aumann and Maschler (1985) shows that in bankruptcy games the nucleolus coincides with the Talmud rule. Depending on whether the sum of the half-claims is above or below the value of the estate, the allocation rule is constrained equal awards or constrained equal losses. Treating the bankrupt agent as a player, therefore, makes much difference. Not only may the bankrupt agent get a positive amount, also the division of the remainder is no longer guided by the application of the constrained equal awards and the constrained equal losses rules, but rather by a truncated proportional rule.

Anderson and Sundaresan (1996) considers a dynamic model with uncertainty in which creditors have the costly option of taking the firm to the bankruptcy court or agree to some debt forgiveness. Yue (2010) models both sovereign default and debt renegotiation in a dynamic equilibrium model, where default may result in the loss of future access to capital markets. What we show is that even without bankruptcy or reputation costs there is a possibility for debt forgiveness, which is obtained by using the threat of paying others. Our argument for debt forgiveness is also complementary to the argument that debt forgiveness would increase the incentive of a debtor to make adjustment efforts to increase the value of the assets (Corden, 1988), to some extent also benefiting the creditors. Our findings can also be seen as a new motive to bail out an organization in a soft budget constraint syndrome (Kornai, 1979).

The rest of the paper is organized as follows. In Section 2 we define and illustrate liability problems and liability games. In Section 3 we analyze the following properties of liability games: additivity, convexity, concavity, superadditivity, and the constant-sum property. In Section 4 we define liability rules and argue in favor of the nucleolus as a liability rule. Section 5 we derive some properties of the payment vectors that result from using the nucleolus. In Section 6 we present conditions under which the nucleolus coincides with a particular generalized truncated proportional rule. Section 7 contains the conclusion.

\section{Liability Problems and Liability Games}

Let $N=\{0,1, \ldots, c\}$ denote the set of agents, where agent 0 is a firm having a set of creditors $C=\{1, \ldots, c\}$ with cardinality $|C|=c \geq 1$. The firm has asset value $A \in \mathbb{R}_{+}$ and liabilities $\ell \in \mathbb{R}_{+}^{C}$, with $\ell_{i} \in \mathbb{R}_{+}$the liability to creditor $i \in C$. The question is how to distribute the asset value of the firm among the creditors and the firm itself.

Definition 2.1. A liability problem is a pair $(A, \ell) \in \mathbb{R}_{+} \times \mathbb{R}_{+}^{C}$. 
Let $\mathcal{L}$ be the class of liability problems.

Given a liability problem $(A, \ell) \in \mathcal{L}$, a payment vector is a vector $x \in \mathbb{R}_{+} \times \mathbb{R}_{+}^{C}$ satisfying liabilities boundedness and efficiency. Liabilities boundedness means that no creditor $i \in C$ receives more than his claim, so $x_{i} \leq \ell_{i}$. Efficiency requires that the sum of the payments should be equal to the asset value: $\sum_{i \in N} x_{i}=A$. Note that non-negativity and efficiency imply that payments to the firm are also bounded from above, $x_{0} \leq A$.

Let $\hat{\ell}$ denote the vector of liabilities truncated by the asset value, that is $\hat{\ell}_{i}=\min \left\{A, \ell_{i}\right\}$ is the truncated liability of creditor $i \in C$. Given a subset of creditors $S \subseteq C$, we will use the notation $\ell(S)=\sum_{i \in S} \ell_{i}$ for the total liabilities of $S$ and $\hat{\ell}(S)=\sum_{i \in S} \hat{\ell}_{i}$ for the total truncated liabilities of $S$. If $\ell(C)>A$, then the asset value is insufficient to honor all the creditors and the firm is said to be insolvent. On the other hand, if $\ell(C) \leq A$, then the firm is solvent. Some of our results depend on whether $\hat{\ell}(C)$ is less than or equal to $A$ or not. The condition $\hat{\ell}(C) \leq A$ is equivalent to either $\ell(C) \leq A$ or there is $i \in C$ such that $\ell_{i}>A$ and $\ell_{j}=0$ for every $j \in C \backslash\{i\}$. Thus $\hat{\ell}(C) \leq A$ means that the firm is solvent or it is insolvent with only one positive liability.

The agents can form coalitions in bargaining over the eventual payment vector. A coalition is an element of $2^{N}$, the collection of all subsets of $N$. The worth of a non-empty coalition $S \in 2^{N}$ is equal to what members of $S$ can guarantee for themselves, irrespective of what outsiders do. It matters greatly whether the firm, player 0 , is a member of $S$ or not. If $0 \in S$, then the firm pays its liabilities to its creditors within the coalition, up to the asset value. Moreover, the coalition can also keep what is left from the asset value after paying all other creditors which is a positive amount if and only if the firm is solvent. If $0 \notin S$, then coalition $S$ can only obtain what is left after the firm has paid its liabilities to creditors not in $S$ in full and at most an amount equal to the value of the liabilities of its members.

A liability problem therefore gives rise to a transferable utility game, which is formally a function $v$ from the set of all coalitions $2^{N}$ to the real numbers with $v(\emptyset)=0$. The set of all transferable utility games with player set $N$ is denoted by $\mathcal{G}$. The next definition formally defines the coalitional worths for the liability game $v$ that results from a liability problem $(\ell, A) \in \mathcal{L}$.

Definition 2.2. Let $(A, \ell) \in \mathcal{L}$ be a liability problem. The induced liability game $v: 2^{N} \rightarrow \mathbb{R}$ is defined by setting, for $S \in 2^{N}$,

$$
v(S)= \begin{cases}\min \{A, \ell(S \backslash\{0\})\}+\max \{A-\ell(C), 0\}, & \text { if } 0 \in S, \\ \min \{\ell(S), \max \{0, A-\ell(C \backslash S)\}\}, & \text { if } 0 \notin S .\end{cases}
$$

Notice that $v(S) \geq 0$ for all $S \in 2^{N}$ and $v(N)=A$. The formulas in Definition 2.2 simplify by specifying them separately for the case where the firm is solvent and the case 
where the firm is insolvent. This is demonstrated in the following theorem, the proof of which is straightforward and therefore omitted.

Theorem 2.3. Let $(A, \ell) \in \mathcal{L}$ be a liability problem. If the firm is solvent, so $\ell(C) \leq A$, then the induced liability game $v: 2^{N} \rightarrow \mathbb{R}$ satisfies, for $S \in 2^{N}$,

$$
v(S)= \begin{cases}A-\ell(C \backslash S), & \text { if } 0 \in S, \\ \ell(S), & \text { if } 0 \notin S .\end{cases}
$$

If the firm is insolvent, so $\ell(C)>A$, then the induced liability game $v: 2^{N} \rightarrow \mathbb{R}$ satisfies, for $S \in 2^{N}$,

$$
v(S)= \begin{cases}\min \{A, \ell(S \backslash\{0\})\}, & \text { if } 0 \in S, \\ \max \{0, A-\ell(C \backslash S)\}, & \text { if } 0 \notin S .\end{cases}
$$

To obtain the worth $v(S)$ for the solvent case when $0 \in S$, observe that in that case $\ell(S \backslash\{0\})+A-\ell(C)=A-\ell(C \backslash S)$. Similarly, to obtain the worth $v(S)$ for the insolvent case when $0 \notin S$, notice that $A-\ell(C \backslash S)<\ell(S)$.

For a game $v \in \mathcal{G}$ and a non-empty coalition $D \in 2^{N}$, the subgame $v^{D}$ with player set $D$ is obtained by restricting $v$ to subsets of $D$. If the game $v$ is induced by a liability problem with an insolvent firm, then for the set of creditors $C$ the subgame $v^{C}$ corresponds to the transferable utility game defined by O'Neill (1982) for standard bankruptcy problems. However, here we also treat the firm as a player. Moreover, we do not restrict attention to the insolvent case. As an illustration of liability games, consider the following examples.

Example 2.4. Consider the liability problem with two creditors, so $N=\{0,1,2\}, A=12$, and $\ell=(6,9)$. The firm in this liability problem is insolvent. The induced liability game $v$ is illustrated in Table 1:

\begin{tabular}{c|ccc|ccc|c}
$S$ & $\{0\}$ & $\{1\}$ & $\{2\}$ & $\{0,1\}$ & $\{0,2\}$ & $\{1,2\}$ & $\{0,1,2\}$ \\
\hline$v(S)$ & 0 & 3 & 6 & 6 & 9 & 12 & 12
\end{tabular}

Table 1: The induced liability game when $N=\{0,1,2\}, A=12, \ell_{1}=6$, and $\ell_{2}=9$.

The worth of coalition $\{1\}$ is equal to 3 , since 3 is the amount that remains for creditor 1 after the firm has paid creditor 2 in full, and corresponds to the amount that creditor 1 can get without the cooperation of the firm. The worth of coalition $\{0,1\}$ is 6 . If the firm cooperates with creditor 1, then together they can achieve 6 by having the firm pay its liability to creditor 1 in full.

In this example, it is easily seen that there is no payment vector $x$ against which no coalition has a complaint. Let $x$ be a payment vector with $x_{0}=0$. Then $v(\{0,1\})=6$ 
and $v(\{0,2\})=9$ implies that $x_{1}+x_{2} \geq 15$ in order for coalitions $\{0,1\}$ and $\{0,2\}$ not to object. Since there are at most 12 units to allocate, this is impossible. Let $x$ be a payment vector with $x_{0}>0$. Then $v(\{1,2\})=12$ implies $x_{0}+x_{1}+x_{2}>12$, leading to a contradiction. The core of the game is empty, and we will see in Section 4 that this is a quite general phenomenon.

The next example illustrates the consequences of raising the asset value in Example 2.4 to 20 .

Example 2.5. Consider the liability problem with two creditors, so $N=\{0,1,2\}, A=20$, and $\ell=(6,9)$. Notice that the firm is solvent. The induced liability game $v$ is illustrated in Table 2. There is exactly one payment vector that does not give rise to objections by

\begin{tabular}{c|c|ccc|ccc|c}
$S$ & $\emptyset$ & $\{0\}$ & $\{1\}$ & $\{2\}$ & $\{0,1\}$ & $\{0,2\}$ & $\{1,2\}$ & $\{0,1,2\}$ \\
\hline$v(S)$ & 0 & 5 & 6 & 9 & 11 & 14 & 15 & 20
\end{tabular}

Table 2: The induced liability game when $N=\{0,1,2\}, A=20, \ell_{1}=6$, and $\ell_{2}=9$.

any coalition: $x=(5,6,9)$. This payment vector is the one that results when the firm pays its two creditors in full and keeps the remainder.

\section{Properties of Liability Games}

In this section, we examine the following properties of liability games.

Definition 3.1. A game $v \in \mathcal{G}$ is:

- Additive if for all $S \in 2^{N}$ we have $v(S)=\sum_{i \in S} v(\{i\})$.

- Constant sum (von Neumann and Morgenstern, 1944) if for all $S \in 2^{N}$ we have $v(S)+v(N \backslash S)=v(N)$.

- Convex Shapley, 1971) if for all $S, T \in 2^{N}$ we have $v(S)+v(T) \leq v(S \cup T)+v(S \cap T)$.

- Concave if for all $S, T \in 2^{N}$ we have $v(S)+v(T) \geq v(S \cup T)+v(S \cap T)$.

- Superadditive if for all $S, T \in 2^{N}$ such that $S \cap T=\emptyset$ we have $v(S)+v(T) \leq v(S \cup T)$.

- Zero-monotonic if for all $i \in N$, for all $S \subseteq N \backslash\{i\}$, we have $v(S)+v(\{i\}) \leq v(S \cup\{i\})$. 
Note that additivity implies the constant sum property and convexity, convexity implies superadditivity, and superadditivity implies zero-monotonicity, but not the other way around.

First, we show that a liability game is additive if and only if the firm is solvent or it is insolvent with only one positive liability.

Theorem 3.2. Given a liability problem $(A, \ell) \in \mathcal{L}$, the induced liability game $v$ is additive if and only if $\hat{\ell}(C) \leq A$. The stand-alone values of an additive liability game are given by $v(\{0\})=A-\hat{\ell}(C)$ and, for every $i \in C, v(\{i\})=\hat{\ell}_{i}$.

\section{Proof.}

The condition $\hat{\ell}(C) \leq A$ is equivalent to either $\ell(C) \leq A$ or there is a single creditor $i \in C$ such that $\ell_{i}>A$ and $\ell_{j}=0$ for all $j \in C \backslash\{i\}$. It holds by Theorem 2.3 that in the former case

$$
v(S)= \begin{cases}A-\ell(C \backslash S), & 0 \in S, \\ \ell(S), & 0 \notin S,\end{cases}
$$

and in the latter case

$$
v(S)= \begin{cases}\min \{A, \ell(S \backslash\{0\})\}=\hat{\ell}(S \backslash\{0\})=\hat{\ell}_{i}, & 0, i \in S, \\ \min \{A, \ell(S \backslash\{0\})\}=0, & 0 \in S, i \in N \backslash S, \\ \max \{0, A-\ell(C \backslash S)\}=A-\hat{\ell}(C \backslash S)=A-0=\hat{\ell}_{i}, & 0 \in N \backslash S, i \in S, \\ \max \{0, A-\ell(C \backslash S)\}=A-\hat{\ell}(C \backslash S)=A-A=0, & 0, i \in N \backslash S .\end{cases}
$$

It is now easily verified that in both cases, $v(\{0\})=A-\hat{\ell}(C), v(\{j\})=\hat{\ell}_{j}$ for all $j \in C$, and $v(S)=\sum_{j \in S} v(\{j\})$ for all $S \subseteq N$, so $v$ is additive.

$(\Rightarrow)$

Suppose $\hat{\ell}(C)>A$. Let $i \in C$ be such that $\ell_{i}>0$. By Theorem 2.3 it holds that

$$
\begin{aligned}
& v(\{0\})=\min \{A, \ell(\emptyset)\}=0, \\
& v(\{i\})=\max \{0, A-\ell(C \backslash\{i\})\} \leq \max \{0, A-\hat{\ell}(C \backslash\{i\})\}<\hat{\ell}_{i},
\end{aligned}
$$

where the strict inequality follows from $\hat{\ell}_{i}>0$ and $\hat{\ell}(C)>A$. At the same time it holds by Theorem 2.3 that

$$
v(\{0, i\})=\min \left\{A, \ell_{i}\right\}=\hat{\ell}_{i},
$$

so $v$ is not additive, yielding a contradiction.

It is easily verified in Examples 2.4 and 2.5 that for every coalition $S \in 2^{N}$ the sum of the worths of coalition $S$ and its complementary coalition $N \backslash S$ is constant and equal to the asset value $A$. We show next that this property holds in all generality. 
Theorem 3.3. Let $(A, \ell) \in \mathcal{L}$ be a liability problem. The induced liability game $v$ is constant sum.

Proof. If $\hat{\ell}(C) \leq A$, then $v$ is additive by Theorem 3.2 and the result follows. We therefore assume $\hat{\ell}(C)>A$, which implies that $\ell(C)>A$. Let some $S \in 2^{N}$ be given. We distinguish two cases: $1.0 \in S$ and $2 . S \subseteq C$.

Case 1. $0 \in S$.

By Theorem 2.3 it holds that

$$
\begin{aligned}
v(S)+v(N \backslash S) & =\min \{A, \ell(S \backslash\{0\})\}+\max \{0, A-\ell(C \backslash(N \backslash S))\} \\
& =\min \{A, \ell(S \backslash\{0\})\}+\max \{0, A-\ell(S \backslash\{0\})\} \\
& =A,
\end{aligned}
$$

where the last equality follows by considering the two cases $\ell(S \backslash\{0\}) \leq A$ and $\ell(S \backslash\{0\})>$ $A$ separately.

Case 2. $S \subseteq C$.

By Theorem 2.3 we have that

$$
\begin{aligned}
v(S)+v(N \backslash S) & =\max \{0, A-\ell(C \backslash S)\}+\min \{A, \ell((N \backslash S) \backslash\{0\})\} \\
& =\max \{0, A-\ell(C \backslash S)\}+\min \{A, \ell(C \backslash S)\} \\
& =A,
\end{aligned}
$$

where the last equality follows by considering the two cases $\ell(C \backslash S) \leq A$ and $\ell(C \backslash S)>A$ separately.

Since the worth of the grand coalition $v(N)$ is equal to the asset value $A$, we know that the sum of the worths of two complementary coalitions is actually equal to $A$.

Liability games feature both concavity and convexity. As we can also see in Examples 2.4 and 2.5, the subgame restricted to the set of creditors is convex. The game restricted to coalitions containing the firm is concave. More precise, in the next result we show that the game $v^{+0}$ on the set of players $C$ defined by

$$
v^{+0}(S)=v(\{0\} \cup S), \quad \emptyset \neq S \subseteq C
$$

is concave.

Theorem 3.4. Let $(A, \ell) \in \mathcal{L}$ be a liability problem and let $v$ be the induced liability game. The subgame $v^{C}$ is convex and the game $v^{+0}$ is concave.

Proof. The subgame $v^{C}$ corresponds to the transferable utility game defined by O'Neill (1982) for standard bankruptcy problems, which is shown to be convex by Curiel, Maschler, and Tijs (1987). 
Consider any $S, T \in 2^{C} \backslash\{\emptyset\}$. We have that

$$
\begin{aligned}
v^{+0}(S)+v^{+0}(T) & =v(\{0\} \cup S)+v(\{0\} \cup T) \\
& =v(N)-v(N \backslash(\{0\} \cup S))+v(N)-v(N \backslash(\{0\} \cup T)) \\
& =v(N)-v^{C}(C \backslash S)+v(N)-v^{C}(C \backslash T) \\
& \left.\geq v(N)-v^{C}((C \backslash S) \cup(C \backslash T))+v(N)-v^{C}(C \backslash S) \cap(C \backslash T)\right) \\
& =v(N)-v^{C}(C \backslash(S \cap T))+v(N)-v^{C}(C \backslash(S \cup T)) \\
& =v(\{0\} \cup(S \cap T))+v(\{0\} \cup(S \cup T)) \\
& =v^{+0}(S \cap T)+v^{+0}(S \cup T),
\end{aligned}
$$

where the second equality uses Theorem 3.3 , the inequality follows from the convexity of $v^{C}$, and the penultimate equality uses Theorem 3.3 again.

The last property we consider in this section is superadditivity. The next result shows that liability games satisfy this property.

Theorem 3.5. Let $(A, \ell) \in \mathcal{L}$ be a liability problem. The induced liability game $v$ is superadditive.

Proof. Let $S, T \in 2^{N}$ be two disjoint coalitions, so $S \cap T=\emptyset$. If $\hat{\ell}(C) \leq A$, then the game is additive by Theorem 3.2 and the result follows. We therefore assume $\hat{\ell}(C)>A$. If $0 \notin S \cup T$, then $v(S)+v(T) \leq v(S \cup T)$ by the convexity of $v^{C}$ as demonstrated in Theorem 3.4 and the result holds, so we only need to consider the situation where $0 \in S \cup T$. Without loss of generality, assume $0 \in S$. By Theorem 2.3 it holds that

$$
\begin{aligned}
v(S) & =\min \{A, \ell(S \backslash\{0\})\}, \\
v(T) & =\max \{0, A-\ell(C \backslash T)\}, \\
v(S \cup T) & =\min \{A, \ell(S \cup T \backslash\{0\})\} .
\end{aligned}
$$

We consider three cases: 1. $A \leq \ell(S \backslash\{0\})$. 2. $A>\ell(S \backslash\{0\})$ and $A \leq \ell(C \backslash T)$. 3 . $A>\ell(S \backslash\{0\})$ and $A>\ell(C \backslash T)$.

Case 1. $A \leq \ell(S \backslash\{0\})$.

It holds that $v(S)=v(S \cup T)=A$. Since $\hat{\ell}(C)>A$, we have that

$$
\ell(C \backslash T) \geq \hat{\ell}(C \backslash T) \geq \hat{\ell}(S \backslash\{0\}) \geq A,
$$

so $v(T)=0$. It follows that $v(S)+v(T) \leq v(S \cup T)$ as desired.

Case 2. $A>\ell(S \backslash\{0\})$ and $A \leq \ell(C \backslash T)$. Since $\ell(C \backslash T) \geq A$, it holds that $v(T)=0$, so

$$
v(S \cup T) \geq v(S)=v(S)+v(T) .
$$


Case 3. $A>\ell(S \backslash\{0\})$ and $A>\ell(C \backslash T)$. It holds that $v(S)=\ell(S \backslash\{0\})$. Since $\ell(C \backslash T)<A$, we have that

$$
v(T)=A-\ell(C \backslash T)<\ell(T),
$$

where the strict inequality follows from $\hat{\ell}(C)>A$. It also holds that

$$
v(T)=A-\ell(C \backslash T) \leq A-\ell(S \backslash\{0\}) .
$$

We find that

$$
\begin{aligned}
v(S)+v(T) & \leq \min \{\ell(S \backslash\{0\})+A-\ell(S \backslash\{0\}), \ell(S \backslash\{0\})+\ell(T)\} \\
& =\min \{A, \ell(S \cup T \backslash\{0\})\}=v(S \cup T) .
\end{aligned}
$$

Since the liability game $v$ is superadditive, it is also zero-monotonic, a property that turns out to be useful in the sequel.

\section{The Nucleolus as a Liability Rule}

A liability rule is a function that assigns a payment vector to each liability problem.

Definition 4.1. A liability rule is a function $f: \mathcal{L} \rightarrow \mathbb{R}_{+}^{N}$ such that, for every $(A, \ell) \in \mathcal{L}$, for every $i \in C, f_{i}(A, \ell) \leq \ell_{i}$ and $\sum_{i \in N} f_{i}(A, \ell)=A$.

A payment vector satisfies non-negativity, liabilities boundedness, and efficiency. A natural way to proceed is to consider the liability game that is induced by the liability problem and to use a liability rule that corresponds to a solution of the liability game. It needs to be shown that the allocation prescribed by the solution is indeed a payment vector.

Let a game $v \in \mathcal{G}$ be given. An allocation is a vector $x \in \mathbb{R}^{N}$ such that $x_{i}$ is the payoff of player $i \in N$. The allocation $x$ yields a total payoff of $x(S)=\sum_{i \in S} x_{i}$ to the members of coalition $S \in 2^{N}$. The allocation $x$ is called efficient if $x(N)=v(N)$ and coalitionally rational if $x(S) \geq v(S)$ for all $S \in 2^{N}$. The allocation $x$ is blocked by coalition $S \in 2^{N}$ if $x(S)<v(S)$.

The set of pre-imputations of a game $v \in \mathcal{G}$ is equal to $I^{*}(v)=\left\{x \in \mathbb{R}^{N} \mid x(N)=\right.$ $v(N)\}$. The addition of individual rationality constraints leads to the set of imputations $I(v)=\left\{x \in I^{*}(v) \mid\right.$ for every $\left.i \in N, x_{i} \geq v(\{i\})\right\}$. The core of the game $v$ (Gillies, 1959), denoted by $\operatorname{Core}(v)$, is the set of efficient and coalitionally rational allocations, $\operatorname{Core}(v)=\left\{x \in I^{*}(v) \mid\right.$ for every $\left.S \in 2^{N}, x(S) \geq v(S)\right\}$. 
We define $\mathcal{N}=2^{N} \backslash\{\emptyset, N\}$ as the collection of all non-empty coalitions that are proper subsets of the grand coalition. The excess $e(S, x)$ of coalition $S \in \mathcal{N}$ at an allocation $x \in \mathbb{R}^{N}$ is given by

$$
e(S, x)=v(S)-x(S)
$$

The excess $e(S, x)$ measures the complaint of coalition $S$ against allocation $x$. The higher the excess $e(S, x)$, the less satisfied coalition $S$ is with $x$. Let $e(x) \in \mathbb{R}^{\mathcal{N}}$ be the vector of excesses at $x$, indexed by $S \in \mathcal{N}$. A pre-imputation $x \in I^{*}(v)$ belongs to Core $(v)$ if and only if for every $S \in \mathcal{N}$ we have that $e(S, x) \leq 0$.

The next result is a corollary of Theorem 3.3 .

Corollary 4.2. Let $(A, \ell) \in \mathcal{L}$ be a liability problem and let $v$ be the induced liability game. For every $S \in \mathcal{N}$, for every pre-imputation $x \in I^{*}(v)$, it holds that $e(S, x)+e(N \backslash S, x)=0$.

Since a liability game is constant sum, it follows that for any coalition and any preimputation the sum of the excesses of the coalition and the complementary coalition is equal to zero. It therefore holds that the core of a liability game is non-empty if and only if all excesses are equal to zero, a very strong condition. Indeed, the following result shows that the core of a liability game is empty if and only if $\hat{\ell}(C)>A$.

Theorem 4.3. Let $(A, \ell) \in \mathcal{L}$ be a liability problem and let $v$ be the induced liability game. If $\hat{\ell}(C) \leq A$, then $\operatorname{Core}(v)=\{(A-\hat{\ell}(C), \hat{\ell})\}$. If $\hat{\ell}(C)>A$, then $\operatorname{Core}(v)=\emptyset$.

Proof. Let $\hat{\ell}(C) \leq A$. Then $v$ is additive by Theorem 3.2. It follows that Core $(v)$ is a singleton, containing the unique imputation $x \in I(v)$ where each player gets his stand-alone value. By Theorem 3.2 it holds that $x_{0}=A-\hat{\ell}(C)$ and, for every $i \in C, x_{i}=\hat{\ell}_{i}$.

Let $\hat{\ell}(C)>A$. Suppose $\operatorname{Core}(v) \neq \emptyset$ and let $x \in \operatorname{Core}(v)$. For every $S \in \mathcal{N}$, it holds that $e(S, x) \leq 0$. By Corollary 4.2 it follows that, for every $S \in \mathcal{N}, e(S, x)=0$. The latter property can only hold if $v$ is additive. By Theorem 3.2 , we have that $\hat{\ell}(C) \leq A$, leading to a contradiction.

In case the firm is solvent, then there is a unique core element. At this core element, the firm pays each of its liabilities in full and keeps the remaining asset value for itself. In case the firm is insolvent and has a single positive liability, there is a unique core element as well. At this core element, the firm pays the creditor with a positive claim an amount equal to $A$ and has a remaining asset value equal to 0 .

In all other cases, the core is empty. There is always a coalition with a complaint irrespective of the pre-imputation chosen. A solution concept that minimizes the complaints of the coalitions then appears as a natural candidate for a liability rule, since it leads to 
allocations as close to the core as possible. This leads us to advocate the nucleolus as introduced in Schmeidler (1969), formally defined next.

The lexicographic order $\preceq_{\text {lex }}$ is the complete order on $\mathbb{R}^{|\mathcal{N}|}$ defined as follows. For $x, y \in \mathbb{R}^{\mathcal{N}}$ it holds that $x \preceq_{\text {lex }} y$ if and only if either $x=y$ or there exists a number $k^{\prime} \in\{1, \ldots,|\mathcal{N}|\}$ such that, for every $k<k^{\prime}, x_{k}=y_{k}$, and $x_{k^{\prime}}<y_{k^{\prime}}$. We have that $x \prec_{\operatorname{lex}} y$ if and only if $x \preceq_{\text {lex }} y$ and $x \neq y$. The coordinate ordering mapping $\theta: \mathbb{R}^{\mathcal{N}} \mapsto \mathbb{R}^{|\mathcal{N}|}$ is defined such that it arranges the coordinates of a vector $x \in \mathbb{R}^{\mathcal{N}}$ in a weakly decreasing order.

Definition 4.4. The nucleolus of a game $v \in \mathcal{G}$ is

$$
\mathrm{Nu}(v)=\{x \in I(v) \mid \text { for every } y \in I(v), \theta(e(x)) \preceq \text { lex } \theta(e(y))\} .
$$

As shown in Schmeidler (1969), the definition of the nucleolus results in a uniquely specified imputation, so the set $\mathrm{Nu}(v)$ is a singleton. From now on, we will therefore treat $\mathrm{Nu}(v)$ as a vector rather than a set. It is the imputation that lexicographically minimizes the ordered excesses. If the core of a game $v \in \mathcal{G}$ is non-empty, then $\mathrm{Nu}(v) \in \operatorname{Core}(v)$. If $v$ is a liability game, then a non-empty core means that the core is a singleton. In this case, either the firm is solvent and the nucleolus then requires the firm to pay its liabilities in full or the firm is insolvent and has a single positive liability on which $A$ is paid, see Theorem 4.3 .

If we replace the set of imputations $I(v)$ by the set of pre-imputations $I^{*}(v)$ in Definition 4.4, then we obtain the pre-nucleolus $\mathrm{Nu}^{*}(v)$ of the game.

Consider the payment vector $x=(1,4,7)$ in Example 2.4, so with a strictly positive payoff of 1 for the firm. The excesses at $(1,4,7)$ for coalitions $S \in \mathcal{N}$ are easily verified to be as follows.

\begin{tabular}{c|ccc|ccc}
$S$ & $\{0\}$ & $\{1\}$ & $\{2\}$ & $\{0,1\}$ & $\{0,2\}$ & $\{1,2\}$ \\
\hline$e(S,(1,4,7))$ & -1 & -1 & -1 & 1 & 1 & 1
\end{tabular}

Table 3: The excesses at $x=(1,4,7)$ in Example 2.4 .

Note that indeed, as stated in Corollary 4.2 , the sum of the excesses of a coalition and its complementary coalition is equal to zero.

The sum of the excesses of the two-player coalitions is equal

$$
\begin{aligned}
v(\{0,1\})+v(\{0,2\})+v(\{1,2\})-2 x(N) & =v(\{0,1\})+v(\{0,2\})+v(\{1,2\})-2 v(N) \\
& =27-24=3 .
\end{aligned}
$$

The highest excess of any of the two-player coalitions is therefore at least equal to 1 and any pre-imputation different from $(1,4,7)$ will lead to some two-player coalition having 
an excess strictly above 1 . It follows that the pre-nucleolus $\mathrm{Nu}^{*}(v)=(1,4,7)$. Since the pre-nucleolus is an imputation, it coincides with the nucleolus.

The firm, even though it is insolvent, gets a strictly positive payment of 1 at the nucleolus. It pays an amount of 4 to creditor 1 , and an amount of 7 to creditor 2, and therefore is allowed to keep 1 unit for itself from the the asset value of its asset value $A=12$. Both creditor 1 and creditor 2 receive 2 units less than their claim on the firm.

We define next the debt forgiveness of a creditor as the difference between the liability towards him and the payment he receives. The truncated debt forgiveness of a creditor is defined as the difference between the truncated liability towards him and the received payment.

Definition 4.5. Let $(A, \ell) \in \mathcal{L}$ be a liability problem and $x \in \mathbb{R}_{+}^{N}$ be a payment vector. The debt forgiveness of creditor $i \in C$ is given by $d_{i}(A, \ell, x)=\ell_{i}-x_{i}$. The truncated debt forgiveness by creditor $i \in C$ is given by $\hat{d}_{i}(A, \ell, x)=\hat{\ell}_{i}-x_{i}=\min \left\{A, \ell_{i}\right\}-x_{i}$.

In Example 2.4, both creditor 1 and creditor 2 have a debt forgiveness of 2 units at the nucleolus. In Example 2.5, all creditors have a debt forgiveness, as well as a truncated debt forgiveness, equal to 0.

The firm is said to receive strong debt forgiveness at a payment vector $x \in \mathbb{R}_{+}^{N}$ if $x_{0}>0$ and there is strictly positive debt forgiveness by at least one of the creditors. If the firm is solvent, it receives strong debt forgiveness if and only if there is strictly positive debt forgiveness by at least one of the creditors. If the firm is insolvent, then it receives strong debt forgiveness if and only if it receives a strictly positive payment.

Another well-known solution for a game $v \in \mathcal{G}$ is the Shapley value (Shapley, 1953) denoted by $\phi(v)$. It is defined by

$$
\phi_{i}(v)=\sum_{S \subseteq N \backslash\{i\}}(v(S \cup\{i\})-v(S)) \frac{|S| !(|N \backslash S|-1) !}{|N| !}, \quad i \in N .
$$

The next result derives the nucleolus for liability problems with $\hat{\ell}(C) \leq A$, thereby generalizing Example 2.5.

Theorem 4.6. Let $(A, \ell) \in \mathcal{L}$ be a liability problem such that $\hat{\ell}(C) \leq A$ and let $v$ be the induced liability game. It holds that $\mathrm{Nu}(v)=\phi(v)=(A-\hat{\ell}(C), \hat{\ell})$, for every $i \in C$, $\hat{d}_{i}(A, \ell, \mathrm{Nu}(v))=0$, and for every $S \in \mathcal{N}, e(S, \mathrm{Nu}(v))=0$.

Proof. Since $v$ is additive by Theorem 3.2 , it is well-known that the nucleolus coincides with the Shapley value and is equal to the unique core element, as given in Theorem 4.3. It is immediate that the truncated debt forgiveness is equal to 0 for every creditor and that all excesses at the nucleolus are equal to zero. 
When $\hat{\ell}(C) \leq A$, then the liability game $v$ is additive, so the nucleolus and the Shapley value coincide. The firm pays every creditor an amount equal to the corresponding truncated liability, so truncated debt forgiveness is equal to zero. In an additive game, all excesses are equal to zero at the nucleolus.

The case $\hat{\ell}(C) \leq A$ means that either the firm is solvent or the firm is insolvent and has only one positive liability. The next result derives the nucleolus for liability problems with an insolvent firm and two positive liabilities, thereby generalizing Example 2.4, and shows it to coincide with the Shapley value.

Theorem 4.7. Let $(A, \ell) \in \mathcal{L}$ be a liability problem with two positive liabilities, say $\ell_{1}$ and $\ell_{2}$, and $\ell(C)>A$. Let $v$ be the induced liability game. It holds that $\mathrm{Nu}(v)=\phi(v)$,

$$
\begin{aligned}
& \mathrm{Nu}_{0}(v)=\frac{2 \hat{\ell_{1}}+2 \hat{\ell_{2}}-2 A}{6}, \\
& \mathrm{Nu}_{1}(v)=\frac{4 A+2 \hat{\ell_{1}}-4 \hat{\ell_{2}}}{6}, \\
& \mathrm{Nu}_{2}(v)=\frac{4 A+2 \hat{\ell_{2}}-4 \hat{\ell_{1}}}{6}, \\
& \mathrm{Nu}_{i}(v)=0, \quad i \in C \backslash\{1,2\} .
\end{aligned}
$$

Both creditors with a positive liability have the same truncated debt forgiveness:

$$
\hat{d}_{1}(A, \ell, \phi(v))=\hat{d}_{2}(A, \ell, \phi(v))=\frac{-4 A+4 \hat{\ell}_{1}+4 \hat{\ell}_{2}}{6} .
$$

Proof. We first consider the liability subgame $\tilde{v}=v_{\{0,1,2\}}$, so we omit the creditors with claims equal to zero. By Theorem 2.3 it follows that $\tilde{v}$ is as presented in Table 4.

\begin{tabular}{c|ccc|ccc|c}
$S$ & $\{0\}$ & $\{1\}$ & $\{2\}$ & $\{0,1\}$ & $\{0,2\}$ & $\{1,2\}$ & $\{0,1,2\}$ \\
\hline$\tilde{v}(S)$ & 0 & $A-\hat{\ell}_{2}$ & $A-\hat{\ell}_{1}$ & $\hat{\ell}_{1}$ & $\hat{\ell}_{2}$ & $A$ & $A$
\end{tabular}

Table 4: The induced liability game when $N=\{0,1,2\}$ and $\hat{\ell}(C)>A$.

The Shapley value is calculated in Table 5. It holds that

$$
\phi(\tilde{v})=\left(\frac{2 \hat{\ell_{1}}+2 \hat{\ell_{2}}-2 A}{6}, \frac{4 A+2 \hat{\ell_{1}}-4 \hat{\ell_{2}}}{6}, \frac{4 A+2 \hat{\ell_{2}}-4 \hat{\ell_{1}}}{6}\right) .
$$

We define $a_{1}=\left(2 A-2 \hat{\ell_{1}}-2 \hat{\ell_{2}}\right) / 6$ and $a_{2}=\left(-2 A+2 \hat{\ell_{1}}+2 \hat{\ell_{2}}\right) / 6$. It holds that $a_{1}<0$ and $a_{2}>0$. Table 6 presents the excesses at $\phi(\tilde{v})$ for coalitions $S \in \mathcal{N}$.

The sum of the excesses of the two-player coalitions is equal to

$$
\begin{aligned}
\tilde{v}(\{0,1\})+\tilde{v}(\{0,2\})+\tilde{v}(\{1,2\})-2 x(N) & =\tilde{v}(\{0,1\})+\tilde{v}(\{0,2\})+\tilde{v}(\{1,2\})-2 \tilde{v}(N) \\
& =\hat{\ell}_{1}+\hat{\ell}_{2}-A .
\end{aligned}
$$

Decreasing any of the excesses of the two-player coalitions below $a_{2}$ would result in an excess above $a_{2}$ at another two-player coalition. The pre-nucleolus of $\tilde{v}$ therefore coincides with the Shapley value. 


\begin{tabular}{c|c|c|c} 
permutation & \multicolumn{3}{|c}{ marginal contribution } \\
\hline & 0 & 1 & 2 \\
\hline $0,1,2$ & 0 & $\hat{\ell}_{1}$ & $A-\hat{\ell}_{1}$ \\
$0,2,1$ & 0 & $A-\hat{\ell}_{2}$ & $\hat{\ell}_{2}$ \\
$1,0,2$ & $\hat{\ell}_{1}+\hat{\ell}_{2}-A$ & $A-\hat{\ell}_{2}$ & $A-\hat{\ell}_{1}$ \\
$1,2,0$ & 0 & $A-\hat{\ell}_{2}$ & $\hat{\ell}_{2}$ \\
$2,0,1$ & $\hat{\ell}_{1}+\hat{\ell}_{2}-A$ & $A-\hat{\ell}_{2}$ & $A-\hat{\ell}_{1}$ \\
$2,1,0$ & 0 & $\hat{\ell}_{1}$ & $A-\hat{\ell}_{1}$ \\
\hline$\phi(\tilde{v})$ & $\frac{2 \hat{\ell}_{1}+2 \hat{\ell}_{2}-2 A}{6}$ & $\frac{4 A+2 \hat{\ell}_{1}-4 \hat{\ell}_{2}}{6}$ & $\frac{4 A+2 \hat{\ell}_{2}-4 \hat{\ell}_{1}}{6}$
\end{tabular}

Table 5: The Shapley value when $N=\{0,1,2\}$ and $\ell(C)>A$.

\begin{tabular}{c|ccc|ccc}
$S$ & $\{0\}$ & $\{1\}$ & $\{2\}$ & $\{0,1\}$ & $\{0,2\}$ & $\{1,2\}$ \\
\hline$e(S, \phi(\tilde{v}))$ & $a_{1}$ & $a_{1}$ & $a_{1}$ & $a_{2}$ & $a_{2}$ & $a_{2}$
\end{tabular}

Table 6: The excesses at the Shapley value when $N=\{0,1,2\}$ and $\ell(C)>A$.

We argue next that the pre-nucleolus of $\tilde{v}$ belongs to the set of imputations $I(\tilde{v})$, so coincides with the nucleolus of $\tilde{v}$. We have that

$$
\begin{aligned}
& \mathrm{Nu}_{0}(\tilde{v})=\frac{2 \hat{\ell_{1}}+2 \hat{\ell_{2}}-2 A}{6}>0=\tilde{v}(\{0\}), \\
& \mathrm{Nu}_{1}(\tilde{v})=\frac{4 A+2 \hat{\ell_{1}}-4 \hat{\ell_{2}}}{6}>A-\hat{\ell}_{2}=\tilde{v}(\{1\}), \\
& \mathrm{Nu}_{2}(\tilde{v})=\frac{4 A+2 \hat{\ell_{2}}-4 \hat{\ell_{1}}}{6}>A-\hat{\ell}_{1}=\tilde{v}(\{2\}),
\end{aligned}
$$

where the strict inequalities follow from the fact that $2 \hat{\ell}_{1}+2 \hat{\ell}_{2}>2 A$.

Since the players in $C \backslash\{1,2\}$ are null players, they get a payoff equal to zero in both the Shapley value and the nucleolus. The payoffs of the other players are the same as in the game $\tilde{v}$.

We finally calculate the level of truncated debt forgiveness at the payment vector $\mathrm{Nu}(v)$. It holds that

$$
\begin{aligned}
& \hat{d}_{1}(A, \ell, \mathrm{Nu}(v))=\hat{\ell}_{1}-\mathrm{Nu}_{1}(v)=\hat{\ell}_{1}-\frac{4 A+2 \hat{\ell}_{1}-4 \hat{\ell}_{2}}{6}=\frac{-4 A+4 \hat{\ell}_{1}+4 \hat{\ell}_{2}}{6}, \\
& \hat{d}_{2}(A, \ell, \mathrm{Nu}(v))=\hat{\ell}_{2}-\mathrm{Nu}_{2}(v)=\hat{\ell}_{2}-\frac{4 A+2 \hat{\hat{\ell}_{2}-4 \hat{\ell}_{1}}}{6}=\frac{-4 A+4 \hat{\ell}_{1}+4 \hat{\ell}_{2}}{6} .
\end{aligned}
$$

As demonstrated in Theorem 4.7, the nucleolus of a liability game with an insolvent firm having two positive liabilities prescribes the same truncated debt forgiveness for both creditors with a positive claim. The firm gets a payoff above its stand-alone value $v(\{0\})$, since $\mathrm{Nu}_{0}(v)=\frac{2 \hat{\ell_{1}}+2 \hat{\ell_{2}}-2 A}{6}>0=v(\{0\})$. The firm is therefore able to keep some of its assets, even though it is insolvent. 
Since the liability game $v$ is constant sum by Theorem 3.3 , the worths of all two-player coalitions are the same in the zero-normalized game. The coincidence of the nucleolus and the Shapley value for the case with $c=2$ then also follows from Yokote, Funaki, and Kamijo (2014), who show that the two-player coalitions having the same worths in the zero-normalized game is a sufficient condition for the coincidence of the nucleolus and the Shapley value in games with three players.

It is easy to find examples showing that the coincidence of Theorem 4.7 does not necessarily hold for liability problems with three or more positive liabilities.

The following result claims that the pre-nucleolus satisfies individual rationality, so the pre-nucleolus coincides with the nucleolus.

Theorem 4.8. Let $(A, \ell) \in \mathcal{L}$ be a liability problem and let $v$ be the induced liability game. For every $i \in N$, it holds that $\mathrm{Nu}_{i}^{*}(v) \geq v(\{i\})$, so $\mathrm{Nu}(v)=\mathrm{Nu}^{*}(v)$.

Proof. The game $v$ is superadditive by Theorem 3.5 and therefore zero-monotonic. It follows from Maschler, Peleg, and Shapley (1979) that, for every $i \in N, \mathrm{Nu}_{i}^{*}(v) \geq v(\{i\})$.

Since the pre-nucleolus coincides with the nucleolus, from now on we will restrict attention to the nucleolus.

By Theorem 2.3 it follows immediately that, for every $i \in N, v(\{i\}) \geq 0$, and

$$
\sum_{i \in N} \mathrm{Nu}_{i}(v)=v(N)=A
$$

This implies that, for every $i \in N, \mathrm{Nu}_{i} \leq A$. The next result shows that the nucleolus also satisfies truncated liabilities boundedness, which implies liabilities boundedness. The nucleolus therefore assigns a payment vector to each liability game.

Theorem 4.9. Let $(A, \ell) \in \mathcal{L}$ be a liability problem and let $v$ be the induced liability game. For every $i \in C$ it holds that $\mathrm{Nu}_{i}(v) \leq \hat{\ell}_{i}$.

Proof. If $\hat{\ell}(C) \leq A$, then $v$ is additive by Theorem 3.2 , so for every $i \in C$ it holds that $\mathrm{Nu}_{i}(v)=v(\{i\})=\hat{\ell}_{i}$. We therefore assume $\hat{\ell}(C)>A$, which implies that $\ell(C)>A$ and hence the liability game is given by the second part of Theorem 2.3. Let $x=\mathrm{Nu}(v)$. Since, for every $i \in N, x_{i} \leq A$, it remains to be shown that $x_{i} \leq \ell_{i}$.

Suppose $i \in C$ is such that $x_{i}>\ell_{i}$. We define $y \in I(v)$ by

$$
\begin{aligned}
& y_{i}=\ell_{i}, \\
& y_{j}=x_{j}+\frac{x_{i}-\ell_{i}}{c}, \quad j \in N \backslash\{i\} .
\end{aligned}
$$

Note that $y \in I(v)$ since $y_{i}=\ell_{i} \geq v(\{i\})$, for every $j \in N \backslash\{i\}, y_{j}>x_{j} \geq v(\{j\})$, and $y(N)=A$. We show next that $\theta(e(y)) \prec_{\text {lex }} \theta(e(x))$, contradicting that $x$ is equal to the nucleolus. 
For every non-empty proper subset $S$ of $N \backslash\{i\}$, consider the pair of excesses

$$
\begin{aligned}
e(S, x) & =v(S)-x(S), \\
e(S \cup\{i\}, x) & =v(S \cup\{i\})-x(S)-x_{i} .
\end{aligned}
$$

Notice that $e(S, x)>e(S \cup\{i\}, x)$ since $v(S \cup\{i\})-v(S) \leq \ell_{i}$ and $x_{i}>\ell_{i}$. The pair of excesses at $y$ is given by

$$
\begin{aligned}
e(S, y) & =v(S)-y(S)=v(S)-x(S)-\frac{|S|\left(x_{i}-\ell_{i}\right)}{c}, \\
e(S \cup\{i\}, y) & =v(S \cup\{i\})-y(S \cup\{i\})=v(S \cup\{i\})-x(S)-\frac{|S|\left(x_{i}-\ell_{i}\right)}{c}-\ell_{i} .
\end{aligned}
$$

We have that $e(S, y)<e(S, x)$ and

$$
e(S, y)-e(S \cup\{i\}, y)=v(S)-v(S \cup\{i\})+\ell_{i} \geq 0
$$

Thus for all the pairs of coalitions that we considered so far, we have lexicographically improved the ordered excesses when replacing the imputation $x$ by the imputation $y$.

The final pair of coalitions to consider is $\{i\}$ and $N \backslash\{i\}$. We have that

$$
e(\{i\}, x)=v(\{i\})-x_{i}=\max \{0, A-\ell(C \backslash\{i\})\}-x_{i}<\max \{0, A-\ell(C \backslash\{i\})\}-\ell_{i}<0,
$$

where the first inequality follows from $x_{i}>\ell_{i}$ and the second inequality from $\ell(C)>A$. It holds that $e(\{i\}, x)<e(\{i\}, y)$ and

$$
e(\{i\}, y)=v(\{i\})-y_{i}=\max \{0, A-\ell(C \backslash\{i\})\}-\ell_{i}<0
$$

By Corollary 4.2, we now get that $e(N \backslash\{i\}, x)=-e(\{i\}, x)>0$ and $e(N \backslash\{i\}, x)>$ $e(N \backslash\{i\}, y)>0$. Thus also this pair of ordered excesses is lexicographically improved when $x$ is replaced by $y$.

We have partitioned $\mathcal{N}$ in pairs and have shown that the ordered excesses of each pair are lexicographically improved when replacing $x$ by $y$. It follows that $e(y) \prec_{\text {lex }} e(x)$, a contradiction to $x$ being the nucleolus. Consequently, it holds that, for every $i \in C, x_{i} \leq \ell_{i}$.

It follows from Theorem 4.9 that we can use the nucleolus as a liability rule.

\section{$5 \quad$ Properties of the Nucleolus}

In this section, we derive properties of the payment vector generated by the nucleolus. We start by analyzing the asset value that the firm is allowed to keep after making its payments. To do so, we first study the vector of excesses in more detail.

Theorem 5.1. Let $(A, \ell) \in \mathcal{L}$ be a liability problem, let $v$ be the induced liability game, and let $x=\mathrm{Nu}(v)$. For every $S \subset T \subset C$, we have: 
1. If $\hat{\ell}(T) \leq A$, then $e(\{0\} \cup S, x) \leq e(\{0\} \cup T, x)$.

2. If $\hat{\ell}(T) \leq A$, then $e(C \backslash S, x) \geq e(C \backslash T, x)$.

3. If $\hat{\ell}(S) \geq A$, then $e(\{0\} \cup S, x) \geq e(\{0\} \cup T, x)$.

4. If $\hat{\ell}(S) \geq A$, then $e(C \backslash S, x) \leq e(C \backslash T, x)$.

Proof. In case $\hat{\ell}(C) \leq A$, all excesses are equal to zero by Theorem 4.6, so the result follows. For the remainder of the proof, we assume $\hat{\ell}(C)>A$.

1. By Theorem 2.3, we have that

$$
\begin{aligned}
e(\{0\} \cup S, x) & =v(\{0\} \cup S)-x(\{0\} \cup S) \\
& =\min \{A, \ell(S)\}-x(\{0\} \cup S)=\hat{\ell}(S)-x(\{0\} \cup S), \\
e(\{0\} \cup T, x) & =v(\{0\} \cup T)-x(\{0\} \cup T) \\
& =\min \{A, \ell(T)\}-x(\{0\} \cup T)=\hat{\ell}(T)-x(\{0\} \cup T),
\end{aligned}
$$

where the last equalities in both equations rely on $\hat{\ell}(S) \leq \hat{\ell}(T) \leq A$. It follows that

$$
e(\{0\} \cup T, x)-e(\{0\} \cup S, x)=\hat{\ell}(T \backslash S)-x(T \backslash S) \geq 0,
$$

where the inequality follows since the nucleolus satisfies truncated liabilities boundedness by Theorem 4.9 .

2. This part follows from part 1 and Corollary 4.2 .

3. By Theorem 2.3, we have that

$$
\begin{aligned}
e(\{0\} \cup S, x) & =v(\{0\} \cup S)-x(\{0\} \cup S) \\
& =\min \{A, \ell(S)\}-x(\{0\} \cup S)=A-x(\{0\} \cup S), \\
e(\{0\} \cup T, x) & =v(\{0\} \cup T)-x(\{0\} \cup T) \\
& =\min \{A, \ell(T)\}-x(\{0\} \cup T)=A-x(\{0\} \cup T),
\end{aligned}
$$

where the last equalities in both equations follow since $A \leq \hat{\ell}(S) \leq \hat{\ell}(T)$. We have that

$$
e(\{0\} \cup S, x)-e(\{0\} \cup T, x)=x(T \backslash S) \geq \sum_{i \in T \backslash S} v(\{i\}) \geq 0
$$

where the first inequality follows from Theorem 4.8 .

4. This part follows from Part 3 and Corollary 4.2 . 
For coalitions containing the firm and with claims totaling to less than the asset value, the excesses at the nucleolus are weakly increasing as the coalitions expand. Since the liability game is constant sum, it then follows that the excesses of the complementary coalitions are weakly decreasing. For coalitions containing the firm with total claims above the asset value, the situation is reversed. The excesses at the nucleolus are weakly decreasing as the coalitions expand and the excesses of the complementary coalitions are weakly increasing.

The result of Theorem 5.1 can be used to show that the coalition of all creditors $C$ has the highest excess at the nucleolus.

Theorem 5.2. Let $(A, \ell) \in \mathcal{L}$ be a liability problem and let $v$ be the induced liability game. For every $S \in \mathcal{N}$ it holds that $e(S, \mathrm{Nu}(v)) \leq e(C, \mathrm{Nu}(v))$.

Proof. In case $\hat{\ell}(C) \leq A$, all excesses are equal to zero by Theorem 4.6, so the result follows. For the remainder of the proof, we assume $\hat{\ell}(C)>A$.

Let $x=\mathrm{Nu}(v)$. It holds that

$$
e(C, x)=v(C)-x(C)=A-\left(x(N)-x_{0}\right)=x_{0} \geq v(\{0\}) \geq 0,
$$

where the first inequality follows from Theorem 4.8 .

We show first that, for every $U \in \mathcal{N}$ such that $U \subset C, e(U, x) \leq e(C, x)$. Let $V=C \backslash U$. If $\hat{\ell}(V) \leq A$, then by taking $S=\emptyset$ and $T=V$ in Part 2 of Theorem 5.1, we have that

$$
e(C, x) \geq e(C \backslash V, x)=e(U, x)
$$

Consider next the case $\hat{\ell}(V)>A$. Take any $i \in U$. By setting $S=V$ and $T=C \backslash\{i\} \supseteq V$ in Part 4 of Theorem 5.1, we get that

$$
e(U, x)=e(C \backslash V, x) \leq e(C \backslash(C \backslash\{i\}), x)=e(\{i\}, x)=v(\{i\})-x_{i} \leq 0,
$$

where the inequality follows from Theorem 4.8. Since $e(C, x) \geq 0$, it also holds that $e(U, x) \leq e(C, x)$ in this case.

Let $T \in \mathcal{N}$ be a coalition with the maximal excess at $x$. Suppose $e(C, x)<e(T, x)$. We have that $0 \in T$ by the first part of the proof. Let $\varepsilon \in(0, e(T, x)-e(C, x))$ and define $y \in I^{*}(v)$ by

$$
\begin{aligned}
& y_{0}=x_{0}+\varepsilon, \\
& y_{i}=x_{i}-\frac{\varepsilon}{c}, \quad i \in C .
\end{aligned}
$$

Let some $S \in \mathcal{N}$ be given. If $S \subseteq C$, then

$$
e(S, y)=e(S, x)+\varepsilon \frac{|S|}{c} \leq e(C, x)+\varepsilon<e(T, x) .
$$


If $0 \in S$, then

$$
e(S, y)=e(S, x)+\varepsilon \frac{|S|-1}{c}-\varepsilon<e(S, x)
$$

It follows that

$$
\max _{S \in \mathcal{N}} e(S, y)<\max _{S \in \mathcal{N}} e(S, x)=e(T, x)
$$

so $\theta(e(y)) \prec_{\text {lex }} \theta(e(x))$, contradicting that $x$ is equal to the pre-nucleolus.

The coalition of all creditors has the maximal excess at the nucleolus. The maximal excess is a non-negative amount.

Next, we show that the firm gets an amount strictly exceeding its stand-alone value if and only if the sum of the truncated liabilities strictly exceeds its asset value.

Theorem 5.3. Let $(A, \ell) \in \mathcal{L}$ be a liability problem and let $v$ be the induced liability game. It holds that $\mathrm{Nu}_{0}(v)>v(\{0\})$ if and only if $\hat{\ell}(C)>A$.

\section{Proof.}

$(\Leftarrow)$ Let $x=\mathrm{Nu}(v)$. It holds that $\ell(C)>A$, so $v(\{0\})=0$ by Theorem 2.3. By Theorem 4.3. Core $(v)=\emptyset$, so there is a coalition with a strictly positive excess at the nucleolus, and by Theorem 5.2 it holds that $e(C, x)>0$. The result follows by observing that $e(C, x)=v(C)-x(C)=A-\left(A-x_{0}\right)=x_{0}$.

$$
(\Rightarrow)
$$

Suppose $\hat{\ell}(C) \leq A$. Then the game $v$ is additive by Theorem 3.2, so $\mathrm{Nu}_{0}(v)=v(\{0\})$, leading to a contradiction. Consequently, it holds that $\hat{\ell}(C)>A$.

When the nucleolus is used as a liability rule, then the firm gets a strictly higher amount than its stand-alone value if and only if the firm is insolvent and has multiple positive liabilities. To put it differently, the firm needs the threat to pay others in order to keep some of its asset value in case of an insolvency. In this case the core of the liability game is empty by Theorem 4.3 and the game is not additive by Theorem 3.2. As a consequence, the firm receives strong debt forgiveness if and only if it is insolvent and has multiple positive liabilities.

We now provide an upper bound on the payment an insolvent firm with multiple positive liabilities receives at the nucleolus. The next theorem shows that it can get at most half of the asset value.

Theorem 5.4. Let $(A, \ell) \in \mathcal{L}$ be a liability problem such that $\hat{\ell}(C) \geq A$ and let $v$ be the induced liability game. It holds that $\mathrm{Nu}_{0}(v) \leq A / 2$. 
Proof. Let $x=\mathrm{Nu}(v)$. Suppose $x_{0}>A / 2$. Let $\varepsilon \in\left(0, x_{0}-A / 2\right)$ and define $y \in I(v)$ by

$$
\begin{aligned}
& y_{0}=x_{0}-\varepsilon, \\
& y_{i}=x_{i}+\frac{\varepsilon}{c}, \quad i \in C .
\end{aligned}
$$

Let some $S \in \mathcal{N}$ be given. If $S \subseteq C$, then

$$
e(S, y)=e(S, x)-\varepsilon \frac{|S|}{c}<e(C, x),
$$

where the inequality follows from Theorem 5.2 .

$$
\begin{aligned}
& \text { If } 0 \in S \text {, then } e(S, x)=v(S)-x(S)<A-A / 2=A / 2 \text { and } \\
& \qquad e(S, y)=e(S, x)-\varepsilon \frac{|S|-1}{c}+\varepsilon \leq e(S, x)+\varepsilon \leq A / 2+\varepsilon<x_{0}=e(C, x) .
\end{aligned}
$$

It follows that

$$
\max _{S \in \mathcal{N}} e(S, y)<e(C, x)=\max _{S \in \mathcal{N}} e(S, x),
$$

so $\theta(e(y)) \prec$ lex $\theta(e(x))$, contradicting that $x$ is equal to the nucleolus.

We show in Section 6 that the firm can get arbitrarily close to $A / 2$ when the nucleolus is used as a liability rule, so the upper bound as provided in Theorem 5.4 is tight.

An interesting question is whether every creditor with a positive claim faces a loss in case the firm is insolvent, so whether there is positive debt forgiveness by each creditor with a positive claim on the firm. The following example shows this is not always the case.

Example 5.5. Consider the liability problem $(\ell, A) \in \mathcal{L}$ with 3 creditors, where $A=10$, $\ell_{1}=1$, and $\ell_{2}=\ell_{3}=8$. Notice that the firm is insolvent. The induced liability game $v$ is depicted in Table 7 .

It is easily verified that the nucleolus of the liability game $v$ is equal to $\mathrm{Nu}(v)=x=$ $(7 / 3,1,10 / 3,10 / 3)$, at which $x_{1}=\ell_{1}=1$. There is no debt forgiveness by creditor 1 , even though the insolvent firm receives strong debt forgiveness and ends up with a strictly positive amount of $x_{0}=7 / 3$. There is positive debt forgiveness by creditors 2 and 3 , $d_{2}(A, \ell, x)=d_{3}(A, \ell, x)=14 / 3$.

The feature in Example 5.5, an insolvent firm paying some of its liabilities in full, can only occur for some of its smaller liabilities. Our last result in this section shows that there is higher debt forgiveness by creditors with higher claims at the nucleolus. We also show that creditors with higher claims receive higher payments at the nucleolus.

Theorem 5.6. Let $(A, \ell) \in \mathcal{L}$ be a liability problem and let $v$ the induced liability game. Let $i, j \in C$ be such that $\ell_{i} \leq \ell_{j}$. At $x=\mathrm{Nu}(v)$ it holds that $x_{i} \leq x_{j}$ and $\ell_{i}-x_{i} \leq \ell_{j}-x_{j}$. 


\begin{tabular}{c|cccc|cccccc}
$S$ & $\{0\}$ & $\{1\}$ & $\{2\}$ & $\{3\}$ & $\{0,1\}$ & $\{0,2\}$ & $\{0,3\}$ & $\{1,2\}$ & $\{1,3\}$ & $\{2,3\}$ \\
\hline$v(S)$ & 0 & 0 & 1 & 1 & 1 & 8 & 8 & 2 & 2 & 9 \\
$x(S)$ & $\frac{7}{3}$ & 1 & $\frac{10}{3}$ & $\frac{10}{3}$ & $\frac{10}{3}$ & $\frac{17}{3}$ & $\frac{17}{3}$ & $\frac{13}{3}$ & $\frac{13}{3}$ & $\frac{20}{3}$ \\
\hline$e(S, x)$ & $-\frac{7}{3}$ & -1 & $-\frac{7}{3}$ & $-\frac{7}{3}$ & $-\frac{7}{3}$ & $\frac{7}{3}$ & $\frac{7}{3}$ & $-\frac{7}{3}$ & $-\frac{7}{3}$ & $\frac{7}{3}$
\end{tabular}

\begin{tabular}{c|cccc}
$S$ & $\{0,1,2\}$ & $\{0,1,3\}$ & $\{0,2,3\}$ & $\{1,2,3\}$ \\
\hline$v(S)$ & 9 & 9 & 10 & 10 \\
$x(S)$ & $\frac{20}{3}$ & $\frac{20}{3}$ & 9 & $\frac{23}{3}$ \\
\hline$e(S, x)$ & $\frac{7}{3}$ & $\frac{7}{3}$ & 1 & $\frac{7}{3}$
\end{tabular}

Table 7: The induced liability game when $N=\{0,1,2,3\}, A=10, \ell_{1}=1$, and $\ell_{2}=\ell_{3}=8$, and the excesses at the nucleolus $x=(7 / 3,1,10 / 3,10 / 3)$.

Proof. Suppose $x_{i}>x_{j}$. We define $y \in I(v)$ by $y_{i}=x_{i}-\varepsilon$ and $y_{j}=x_{j}+\varepsilon$, where $\varepsilon$ is chosen sufficient small such that $y_{i}>y_{j}$. The other components of $y$ are set equal to the corresponding components of $x$. For coalitions $S \in \mathcal{N}$ such that both $i$ and $j$ belong to $S$ or both $i$ and $j$ belong to $C \backslash S$, it clearly holds that $e(S, x)=e(S, y)$.

Take some $S \subseteq N \backslash\{i, j\}$. We demonstrate that

$$
\max \{e(S \cup\{i\}, x), e(S \cup\{j\}, x)\}>\max \{e(S \cup\{i\}, y), e(S \cup\{j\}, y)\} .
$$

It holds that

$$
\begin{aligned}
\max \{e(S \cup\{i\}, x), e(S \cup\{i\}, x)\} & =\max \{v(S \cup\{i\})-x(S \cup\{i\}), v(S \cup\{j\})-x(S \cup\{j\})\} \\
& =v(S \cup\{j\})-x(S \cup\{j\})\},
\end{aligned}
$$

where the last equality follows from $v(S \cup\{i\}) \leq v(S \cup\{j\})$ and $x_{i}>x_{j}$.

It holds that

$$
\begin{aligned}
\max \{e(S \cup\{i\}, y), e(S \cup\{i\}, y)\} & =\max \{v(S \cup\{i\})-y(S \cup\{i\}), v(S \cup\{j\})-y(S \cup\{j\})\} \\
& =v(S \cup\{j\})-y(S \cup\{j\})\}, \\
& =v(S \cup\{j\})-x(S \cup\{j\})-\varepsilon\},
\end{aligned}
$$

where the second equality follows from $v(S \cup\{i\}) \leq v(S \cup\{j\})$ and $y_{i}>y_{j}$.

It follows that $\theta(e(y)) \prec_{\text {lex }} \theta(e(x))$, contradicting that $x$ is equal to the nucleolus. Consequently, it holds that $x_{i} \leq x_{j}$.

Suppose $\ell_{i}-x_{i}>\ell_{j}-x_{j}$. We define $y \in I(v)$ by $y_{i}=x_{i}+\varepsilon$ and $y_{j}=x_{j}-\varepsilon$, where $\varepsilon$ is chosen sufficient small such that $\ell_{i}-y_{i}>\ell_{j}-y_{j}$. The other components of $y$ are set equal to the corresponding components of $x$. For coalitions $S \in \mathcal{N}$ such that both $i$ and $j$ belong to $S$ or both $i$ and $j$ belong to $C \backslash S$, it clearly holds that $e(S, x)=e(S, y)$. 
Take some $S \subseteq N \backslash\{i, j\}$. We demonstrate that

$$
\max \{e(S \cup\{i\}, x), e(S \cup\{j\}, x)\}>\max \{e(S \cup\{i\}, y), e(S \cup\{j\}, y)\} .
$$

It holds that

$$
\begin{aligned}
\max \{e(S \cup\{i\}, x), e(S \cup\{i\}, x)\} & =\max \{v(S \cup\{i\})-x(S \cup\{i\}), v(S \cup\{j\})-x(S \cup\{j\})\} \\
& =v(S \cup\{i\})-x(S \cup\{i\})\},
\end{aligned}
$$

where the last equality follows from $v(S \cup\{i\})-v(S \cup\{j\}) \geq \ell_{i}-\ell_{j}$ and $\ell_{i}-x_{i}>\ell_{j}-x_{j}$.

It holds that

$$
\begin{aligned}
\max \{e(S \cup\{i\}, y), e(S \cup\{i\}, y)\} & =\max \{v(S \cup\{i\})-y(S \cup\{i\}), v(S \cup\{j\})-y(S \cup\{j\})\} \\
& =v(S \cup\{i\})-y(S \cup\{i\})\}, \\
& =v(S \cup\{i\})-x(S \cup\{i\})-\varepsilon\},
\end{aligned}
$$

where the second equality follows from $v(S \cup\{i\})-v(S \cup\{j\}) \geq \ell_{i}-\ell_{j}$ and $\ell_{i}-y_{i}>\ell_{j}-y_{j}$.

It follows that $\theta(e(y)) \prec_{\text {lex }} \theta(e(x))$, contradicting that $x$ is equal to the nucleolus. Consequently, it holds that $\ell_{i}-x_{i} \leq \ell_{j}-x_{j}$.

Theorem 5.6 implies using the nucleolus as a liability rule leads to the same payment to creditors with identical claims. In terms of debt forgiveness, we have seen in Example 5.5 that there is no debt forgiveness by creditors with low claims even when the firm is insolvent. Theorem 5.6 generalizes this insight and implies that there is a threshold such that there is no debt forgiveness by creditors with claims below the threshold and positive debt forgiveness by creditors with claims above the threshold.

\section{The Generalized Proportional Rule}

In principe, it is possible to compute the nucleolus by solving a linear programming problem. However, the size of the linear programming problem is related to the number of coalitions, which increases exponentially in the number of players. For instance, in a game with $n$ players, Owen (1974) presents a linear programming formulation to compute the nucleolus that involves $2^{n+1}+n$ variables and $4^{n}+1$ constraints. In this section, we argue that for liability problems there is a relation between the nucleolus and the so-called generalized truncated proportional rule and we have a closed from solution for the latter.

A well-known liability rule in bankruptcy situations is the proportional rule. Under the proportional rule, in case of an insolvency, the entire asset value is distributed to the creditors in proportion to their liabilities. The insolvent agent is then left without any assets. We define the generalized proportional rule by making payments in proportion to the liabilities, but by allowing for a non-negative payment to the firm even in case of insolvency. 
Definition 6.1. The liability rule $p: \mathcal{L} \rightarrow \mathbb{R}_{+}^{N}$ is a generalized proportional rule if for every $(A, \ell) \in \mathcal{L}$ there is $\lambda \in \mathbb{R}_{+}$such that for every $i \in C$ it holds that $p_{i}(A, \ell)=\lambda \ell_{i}$.

Recall that by definition of a liability rule it holds that $0 \leq p_{0}(A, \ell)$, for every $i \in C$, $0 \leq p_{i}(A, \ell) \leq \ell_{i}$, and $\sum_{i \in N} p_{i}(A, \ell)=A$. Given the amount $p_{0}(A, \ell)$ the firm is allowed to keep for itself, the payments to the creditors are uniquely determined under the generalized proportional rule.

The truncated proportional rule distributes the entire asset value in proportion to the truncated liabilities. The generalized truncated proportional rule does the same, but allows the insolvent agent to keep some of its asset value.

Definition 6.2. The liability rule $\hat{p}: \mathcal{L} \rightarrow \mathbb{R}_{+}^{N}$ is a generalized truncated proportional rule if for every $(A, \ell) \in \mathcal{L}$ there is $\lambda \in \mathbb{R}_{+}$such that for every $i \in C$ it holds that $p_{i}(A, \ell)=\lambda \hat{\ell}_{i}$.

Again, once the amount $\hat{p}_{0}(A, \ell) \in[0, A]$ the firm is allowed to keep has been decided upon, the payments to the creditors are uniquely determined under the generalized truncated proportional rule. Let $\hat{p}^{*}: \mathcal{L} \rightarrow \mathbb{R}_{+}^{N}$ denote the generalized truncated proportional rule obtained when

$$
\hat{p}_{0}^{*}(A, \ell)= \begin{cases}A-\hat{\ell}(C), & \text { if } \hat{\ell}(C) \leq A, \\ \frac{(\hat{\ell}(C)-A) A}{2 \hat{\ell}(C)-A}, & \text { if } \hat{\ell}(C)>A .\end{cases}
$$

It is easily verified that $\hat{p}_{0}^{*}(A, \ell) \in[0, A]$.

We first study liability problems with $\hat{\ell}(C) \leq A$, so the firm is either solvent or has a single positive liability. The following result is an immediate corollary of Theorem 4.6 .

Corollary 6.3. Let $(A, \ell) \in \mathcal{L}$ be a liability problem such that $\hat{\ell}(C) \leq A$ and let $v$ be the induced liability game. It holds that $\mathrm{Nu}(v)=\hat{p}^{*}(A, \ell)$.

We next turn to the case of an insolvent firm with multiple positive liabilities.

For a given liability problem $(A, \ell) \in \mathcal{L}$, let $\tilde{C}$ be the set of creditors with a liability strictly in between 0 and $A$, so $\tilde{C}=\left\{i \in C \mid 0<\ell_{i}<A\right\}$, and denote the cardinality of $\tilde{C}$ by $\tilde{c}$. For each non-empty $S \subseteq \tilde{C}$, let $\tilde{m}(S) \in \mathbb{R}^{\tilde{C}}$ denote the membership vector of $S$, so $\tilde{m}_{i}(S)=1$ if $i \in S$ and $\tilde{m}_{i}(S)=0$ if $i \in \tilde{C} \backslash S$. Let $\tilde{\mathcal{S}}$ be a collection of non-empty coalitions of creditors in $\tilde{C}$, so for every $S \in \tilde{\mathcal{S}}$ it holds that $\emptyset \neq S \subseteq \tilde{C}$. The collection $\tilde{\mathcal{S}}$ is independent if $\{\tilde{m}(S) \mid S \in \tilde{\mathcal{S}}\}$ is a set of independent vectors. The collection $\tilde{\mathcal{S}}$ is balanced if there are weights $\left(\lambda_{S}\right)_{S \in \tilde{\mathcal{S}}} \in \mathbb{R}_{++}^{\tilde{\mathcal{S}}}$ such that $\sum_{S \in \tilde{\mathcal{S}}} \lambda_{S} \tilde{m}(S)=\tilde{m}(\tilde{C})$.

Assumption 6.4. The liability problem $(A, \ell) \in \mathcal{L}$ is such that $\hat{\ell}(C)>A$ and there exists an independent and balanced collection of coalitions $\tilde{\mathcal{S}}$ with $|\tilde{\mathcal{S}}|=\tilde{c}$ such that for every $S \in \tilde{\mathcal{S}}$ we have that $\ell(S)=A$. 
As an illustration, the following example presents a number of cases where Assumption 6.4 is satisfied.

\section{Example 6.5.}

Case 1. The liability problem $(A, \ell) \in \mathcal{L}$ is such that $\hat{\ell}(C)>A$ and for every $i \in C, \ell_{i}=0$ or $\ell_{i} \geq A$. It holds that $\tilde{C}=\emptyset$ and Assumption 6.4 is trivially satisfied.

Case 2. The liability problem $(A, \ell) \in \mathcal{L}$ has exactly three creditors with a claim in $(0, A)$, without loss of generality, $\tilde{C}=\{1,2,3\}$, and $\left(\ell_{1}, \ell_{2}, \ell_{3}\right)=\left(\frac{1}{2} A, \frac{1}{2} A, \frac{1}{2} A\right)$. Then Assumption 6.4 is satisfied with $\tilde{\mathcal{S}}=\{\{1,2\},\{1,3\},\{2,3\}\}$ and $\lambda_{S}=1 / 2$ for all $S \in \tilde{\mathcal{S}}$.

Case 3. The liability problem $(A, \ell) \in \mathcal{L}$ has exactly four creditors with a claim in $(0, A)$, without loss of generality, $\tilde{C}=\{1,2,3,4\}$, and $\left(\ell_{1}, \ell_{2}, \ell_{3}, \ell_{4}\right)=\left(\frac{1}{3} A, \frac{1}{3} A, \frac{1}{3} A, \frac{2}{3} A\right)$. Then Assumption 6.4 is satisfied with $\tilde{\mathcal{S}}=\{\{1,4\},\{2,4\},\{3,4\},\{1,2,3\}\}$ and $\lambda_{\{1,4\}}=$ $\lambda_{\{2,4\}}=\lambda_{\{3,4\}}=1 / 3$ and $\lambda_{\{1,2,3\}}=2 / 3$.

The next result shows that under Assumption 6.4, the nucleolus corresponds to the payment generated by the generalized truncated proportional rule $\hat{p}^{*}$.

Theorem 6.6. Let $(A, \ell) \in \mathcal{L}$ be a liability problem satisfying Assumption 6.4 and let $v$ be the induced liability game. It holds that $\mathrm{Nu}(v)=\hat{p}^{*}(A, \ell)$, so

$$
\begin{aligned}
\mathrm{Nu}_{0}(v) & =\frac{(\hat{\ell}(C)-A) A}{2 \hat{\ell}(C)-A}, \\
\mathrm{Nu}_{i}(v) & =\frac{A}{2 \hat{\ell}(C)-A} \hat{\ell}_{i}, \quad \text { for all } i \in C .
\end{aligned}
$$

Proof. Let $x=\hat{p}^{*}(A, \ell)$. We show that $x$ is the nucleolus of $v$.

We show first that the excesses of all coalitions are less than or equal to $x_{0}$.

Let some $S \in \mathcal{N}$ be given. We distinguish the cases $0 \in S$ and $0 \notin S$.

Case $1.0 \in S$.

We have that

$$
\begin{aligned}
e(S, x) & =v(S)-x(S) \\
& =\min \{A, \ell(S \backslash\{0\})\}-x_{0}-\frac{A}{2 \hat{\ell}(C)-A} \hat{\ell}(S \backslash\{0\}) \\
& =\min \{A, \hat{\ell}(S \backslash\{0\})\}-x_{0}-\frac{A}{2 \hat{\ell}(C)-A} \hat{\ell}(S \backslash\{0\}) \\
& =\left(1-\frac{A}{2 \hat{\ell}(C)-A}\right) \min \{A, \hat{\ell}(S \backslash\{0\})\}-x_{0} \\
& \leq\left(1-\frac{A}{2 \hat{\ell}(C)-A}\right) A-x_{0} \\
& =x_{0},
\end{aligned}
$$

where the second equality follows from Theorem 2.3 .

Case 2. $0 \notin S$.

By Theorem 2.3 it holds that

$$
e(S, x)=v(S)-x(S)=\max \{0, A-\ell(C \backslash S)\}-\left(A-x_{0}-x(C \backslash S)\right) .
$$


If $A-\ell(C \backslash S) \geq 0$, then the right-hand side of the equation above equals

$$
A-\ell(C \backslash S)-A+x_{0}+x(C \backslash S) \leq x_{0} .
$$

Otherwise, $A-\ell(C \backslash S)<0$ and the right-hand side is equal to

$$
-A+x_{0}+x(C \backslash S) \leq x_{0} .
$$

Let $\tilde{\mathcal{S}}$ be an independent and balanced collection of coalitions with $|\tilde{\mathcal{S}}|=\tilde{c}$ such that for all $S \in \tilde{\mathcal{S}}$ we have that $\ell(S)=A$ and let $\left(\lambda_{S}\right)_{S \in \tilde{\mathcal{S}}} \in \mathbb{R}_{+}^{\tilde{\mathcal{S}}}$ be the corresponding vector of balancing weights. We define $\bar{C}=\left\{i \in C \mid \ell_{i}>0\right\}$ as the set of creditors with a positive claim and denote its cardinality by $\bar{c}$. We define $\overline{\mathcal{S}}=\tilde{\mathcal{S}} \cup\{\{i\} \mid i \in \bar{C} \backslash \tilde{C}\}$ as the collection of coalitions in $\tilde{\mathcal{S}}$ together with the singletons whose members have a claim greater than or equal to $A$. For a non-empty subset $S$ of $\bar{C}$, we define the membership vector $\bar{m}(S) \in \mathbb{R}^{\bar{C}}$ by $\bar{m}_{i}(S)=1$ if $i \in S$ and $\bar{m}_{i}(S)=0$ if $i \in \bar{C} \backslash S$. We denote $\bar{m}(\bar{C})$ by $\mathbb{1}$.

Since the set $\{\tilde{m}(S) \mid S \in \tilde{\mathcal{S}}\}$ is a set of independent vectors of cardinality $\tilde{c}$ and $\{\bar{m}(\{i\}) \mid i \in \bar{C} \backslash \tilde{C}\}$ is a set of unit vectors with coordinate one at a player not being part of some $S \in \tilde{\mathcal{S}}$, the set $\{\bar{m}(S) \mid S \in \overline{\mathcal{S}}\}$ is a set of independent vectors of cardinality $\bar{c}$. For $i \in \bar{C} \backslash C$, we define $\lambda_{\{i\}}=1$. We have that

$$
\sum_{S \in \overline{\mathcal{S}}} \lambda_{S} \bar{m}(S)=\mathbb{1}
$$

or in matrix notation, with $M$ being the matrix with columns equal to $\bar{m}(S)$ for $S \in \overline{\mathcal{S}}$,

$$
M \lambda=\mathbb{1} .
$$

It holds that

$$
\sum_{S \in \overline{\mathcal{S}}} \lambda_{S}>1
$$

since by Assumption $6.4 \hat{\ell}(C)>A$, so $\bar{c} \geq 2$, and the set $\overline{\mathcal{S}}$ therefore contains at least two coalitions, whereas the sum of the weights over the members of each coalition is equal to 1 .

Let some $S \in \overline{\mathcal{S}}$ be given. We have by Theorem 2.3 that $v(\{0\} \cup S)=\min \{A, \ell(S)\}=A$, where the last equality follows from Assumption 6.4 if $S \in \tilde{\mathcal{S}}$ and from $\ell_{i} \geq A$ if $S=\{i\}$ for $i \in \bar{C} \backslash \tilde{C}$. It follows that

$$
\begin{aligned}
e(\{0\} \cup S, x) & =v(\{0\} \cup S)-x(\{0\} \cup S) \\
& =A-x_{0}-\frac{A}{2 \hat{\ell}(C)-A} \hat{\ell}(S) \\
& =A-\frac{(\hat{\ell}(C)-A) A}{2 \hat{\ell}(C)-A}-\frac{A}{2 \hat{\ell}(C)-A} A \\
& =\frac{(\hat{\ell}(C)-A) A}{2 \hat{\ell}(C)-A}=x_{0} .
\end{aligned}
$$


For the coalition of all creditors, we have that

$$
e(C, x)=v(C)-x(C)=A-\left(A-x_{0}\right)=x_{0}
$$

as well.

Let $y=\mathrm{Nu}(v)$ and suppose $y \neq x$. We have that $\theta(e(y)) \prec_{\text {lex }} \theta(e(x))$. From

$$
e(C, y)=v(C)-y(C)=A-A+y_{0}=y_{0},
$$

it follows that $y_{0} \leq x_{0}$.

For every $S \in \overline{\mathcal{S}}$ it holds that

$$
\begin{aligned}
e(\{0\} \cup S, y) & =v(\{0\} \cup S)-y(\{0\} \cup S)=A-y(\{0\} \cup S) \\
& \leq x_{0}=e(\{0\} \cup S, x)=A-x(\{0\} \cup S) .
\end{aligned}
$$

It follows that, for every $S \in \overline{\mathcal{S}}, x_{0}+x(S) \leq y_{0}+y(S)$, or in matrix notation

$$
x_{0} \mathbb{1}+M^{\top} x_{\bar{C}} \leq y_{\bar{C}} \mathbb{1}+M^{\top} y_{\bar{C}} .
$$

Consider first the case $x_{0}=y_{0}$. Since $x \neq y$ and $M$ has full rank, we have that

$$
M^{\top} x_{\bar{C}}<M^{\top} y_{\bar{C}} .
$$

Taking the inner product with the vector of balancing weights $\lambda$, we obtain

$$
\mathbb{1}^{\top} x_{\bar{C}}=\lambda^{\top} M^{\top} x_{\bar{C}}<\lambda^{\top} M^{\top} y_{\bar{C}}=\mathbb{1}^{\top} y_{\bar{C}} .
$$

For every $i \in C$ such that $\ell_{i}=0$, it holds that $x_{i}=y_{i}=0$ since $x$ and $y$ are payment vectors. Also, $x_{0}=y_{0}$ and $x(N)=y(N)$, so we find that $\mathbb{1}^{\top} x_{\bar{C}}=\mathbb{1}^{\top} y_{\bar{C}}$, leading to a contradiction with (6.3).

Consider next the case $y_{0}<x_{0}$. We take the inner product of $(6.2)$ with the vector of balancing weights $\lambda$. Since

$$
\begin{aligned}
& \lambda^{\top} M^{\top} x_{\bar{C}}=\mathbb{1}^{\top} x_{\bar{C}}=\mathbb{1}^{\top} x_{C}=A-x_{0}, \\
& \lambda^{\top} M^{\top} y_{\bar{C}}=\mathbb{1}^{\top} y_{\bar{C}}=\mathbb{1}^{\top} y_{C}=A-y_{0},
\end{aligned}
$$

we get

$$
x_{0} \lambda^{\top} \mathbb{1}+A-x_{0} \leq y_{0} \lambda^{\top} \mathbb{1}+A-y_{0},
$$

which is equivalent to

$$
\left(x_{0}-y_{0}\right)\left(\lambda^{\top} \mathbb{1}-1\right) \leq 0 .
$$


Since $x_{0}>y_{0}$ and $\lambda^{\top} \mathbb{1}=\sum_{S \in \overline{\mathcal{S}}} \lambda_{S}>1$ by 6.1, we obtain a contradiction. Consequently, it holds that $x=y$ as was to be shown.

The intuition behind Theorem 6.6 is as follows. By Theorem 5.2, the coalition of all creditors $C$ has the highest excess among all coalitions at the nucleolus. By Theorem 5.1, a coalition $S$ such that $\hat{\ell}(S)=A$ is such that at the nucleolus the excess of coalition $\{0\} \cup S$ is greater than or equal to the excess of coalition $\{0\} \cup T$ if $S$ contains $T$ or $T$ contains $S$. Assumption 6.4 ensures that we can find $\tilde{c}$ coalitions $S$ that are balanced and independent such that $\ell(S)=\hat{\ell}(S)=A$. Together with singleton coalitions consisting of $i \in C$ such that $\ell_{i} \geq A$, this leads to $\bar{c}$ coalitions $S$ that are balanced and independent such that $\hat{\ell}(S)=A$, where $\bar{c}$ is the number of positive liabilities. The system of equations that results from equating the excess of all these coalitions with the excess of coalition $C$ and specifying payments of zero for liabilities equal to zero has full rank and therefore a unique solution. The solution is then shown to be the nucleolus.

Let $(A, \ell) \in \mathcal{L}$ be a liability problem such that $\ell_{i} \geq A$ for every $i \in C$. Case 1 in Example 6.5 is satisfied, so the nucleolus $\mathrm{Nu}(v)$ of the induced liability game is equal to $\hat{p}^{*}(A, \ell)$. We have that

$$
\hat{p}_{0}^{*}(A, \ell)=\frac{(\hat{\ell}(C)-A) A}{2 \hat{\ell}(C)-A}=\frac{(c A-A) A}{2 c A-A}=\frac{(c-1) A}{2 c-1} .
$$

Theorem 5.4 states that the payment received by an insolvent firm is at most equal to half of the asset value. The expression above shows that debt forgiveness can get arbitrarily close to $A / 2$ for large values of $c$.

Assumption 6.4 is not satisfied in Example 2.4. According to the generalized truncated proportional rule $\hat{p}^{*}$, the payoff vector should be $(2,4,6)$, whereas the nucleolus assigns $(1,4,7)$. The reason is that there are no coalitions $S$ in Example 2.4 such that $\ell(S)=A$. However, in many examples it is possible to find a collection of coalitions that is balanced and independent such that $\ell(S)$ is approximately equal to $A$ for every coalition $S$ in the collection. The generalized truncated proportional rule $\hat{p}^{*}$ is therefore approximately equal to the nucleolus in many examples. Our last example demonstrates this point for the case where the firm is insolvent and all liabilities are the same.

Example 6.7. Consider a liability problem $(\ell, A) \in \mathcal{L}$ where all liabilities are identical and equal to $a<A$. We assume the firm is insolvent, so $c a>A$. Let $k \in \mathbb{N}$ be such that $k a \leq A$ and $(k+1) a>A$. If $S \subset C$ is a coalition with $k$ creditors, then $v(\{0\} \cup S)=k a$ and if $T \subseteq C$ is a coalition with $k+1$ creditors than $v(\{0\} \cup T)=A$. We denote the nucleolus $\mathrm{Nu}(v)$ of the liability game $v$ by $x$. Since all liabilities are identical, all creditors 
receive the same payoff, $x_{1}$. We have that

$$
\begin{aligned}
& e(\{0\} \cup S, x)=k a-x_{0}-k x_{1}, \\
& e(\{0\} \cup T, x)=A-x_{0}-(k+1) x_{1} .
\end{aligned}
$$

It holds that $v(C)=A$ and $e(C, x)=x_{0}$. It holds that $k a-x_{0}-k x_{1} \geq A-x_{0}-(k+1) x_{1}$ if and only if $x_{1} \geq A-k a$.

We solve for

$$
\begin{aligned}
& k a-x_{0}-k x_{1}=x_{0}, \\
& x_{0}+c x_{1}=A,
\end{aligned}
$$

and find

$$
\begin{aligned}
& x_{0}=\frac{c k a-k A}{2 c-k}, \\
& x_{1}=\frac{2 A-k a}{2 c-k} .
\end{aligned}
$$

The condition $x_{1} \geq A-k a$ is then equivalent to

$$
k a \frac{2 c-k-1}{2 c-k-2} \geq A
$$

This condition is clearly satisfied whenever $k a=A$. In that case, we get exactly the payment vector of the generalized truncated proportional rule $\hat{p}^{*}(A, \ell)$ of Theorem 6.6, so

$$
\begin{aligned}
& x_{0}=\frac{(c-k) A}{2 c-k}, \\
& x_{1}=\frac{A}{2 c-k} .
\end{aligned}
$$

To the extent that $k a$ is almost equal to $A$, we get that the nucleolus is almost equal to the generalized truncated proportional rule $\hat{p}^{*}(A, \ell)$.

In case $k a(2 c-k-1)<A(2 c-k-2)$, we find the nucleolus by solving

$$
\begin{aligned}
& A-x_{0}-(k+1) x_{1}=x_{0}, \\
& x_{0}+c x_{1}=A,
\end{aligned}
$$

and find

$$
\begin{aligned}
& x_{0}=\frac{c A-(k+1) A}{2 c-k-1}, \\
& x_{1}=\frac{A}{2 c-k-1} .
\end{aligned}
$$

Again, to the extent that $(k+1) a$ is almost equal to $A$, we get that the nucleolus is almost equal to the payment vector of the generalized truncated proportional rule $\hat{p}^{*}(A, \ell)$. 


\section{Conclusion}

A liability problem consists of a firm with a certain asset value having liabilities towards a group of creditors. We study the allocation of the asset value of the firm among the creditors using transferable utility games and, contrary to the large body of game-theoretic work on bankruptcy games, treat the firm as a player. A major advantage of using transferable utility games is that we can avoid the specification of the fine details of the negotiation process.

We show that the core of the game is non-empty if and only if the firm is insolvent and has multiple positive liabilities. This means that in most situations with insolvency, irrespective of the allocation of the asset value of the firm to the creditors, there is always some coalition that has a complaint. We are therefore interested in payment vectors that are generated by the nucleolus of liability game as these are payment vectors that minimize the complaints coalitions can have. We show that the nucleolus assigns a payoff to the firm above the firm's stand-alone value if and only if it is insolvent and has multiple positive liabilities. In these cases, the firm is allowed to keep part of its asset value, and there is, therefore, debt forgiveness by some of its creditors. The reason for debt forgiveness is that the firm in a liability game has the "threat to pay others."

We show that an insolvent firm ends up with at most one half of its asset value and that this upper bound is tight. We also show that creditors with higher claims get higher payments, but they also have higher debt forgiveness. It follows that there is a threshold on the size of the liabilities such that below the threshold there is no debt forgiveness and above the threshold there is positive debt forgiveness. In many cases, this threshold is equal to zero, but we show that there are examples where the threshold is positive.

In general, there is no closed-form solution for the liability rule that is generated by the nucleolus. However, we provide conditions under which it coincides with a generalized truncated proportional rule. We have a closed-form solution for the amount of the asset value that the firm is allowed to keep. The remainder of the asset value is allocated to the creditors in proportion to their claim on the firm, where that claim is truncated by the firm's asset value.

In the game-theoretic literature on bankruptcy games, the bankrupt agent is not treated as a player, but rather as an exogenous estate. As shown by Aumann and Maschler (1985), the nucleolus corresponds to the constrained equal awards rule if the value of the estate is below the sum of the half-claims and the constrained equal losses rule if it is above. It is striking that the case where the bankrupt agent is a player makes such a big difference in the allocation of the asset value.

We believe there to be quite some potential for future research on liability games. One question is whether the generalized truncated proportional rule we find can be axiomatized. 
One may also study the properties of solutions different from the nucleolus. We have taken the theory of transferable utility games to bypass the exact modeling of the negotiation process. It would also be interesting to study the equilibria of non-cooperative bargaining models. The model can be generalized and extended in many different ways. One possible extension is to go from a single bankrupt agent with creditors to the case where the creditors are agents themselves and may also have solvency issues. The model then becomes an extension of the seminal model of Eisenberg and Noe (2001) to study bankruptcy in financial networks.

\section{References}

Anderson, R.W., and S. Sundaresan, 1996, "Design and valuation of debt contracts," Review of Financial Studies, 9, 37-68.

Arslanalp, S., and P.B. Henry, 2005, "Is debt relief efficient?," Journal of Finance, 60, $1017-1051$

Aumann, R.J., and M. Maschler, 1985, "Game theoretic analysis of a bankruptcy problem from the Talmud," Journal of Economic Theory, 36, 195-213.

Benjamin, D., and M.L.J. Wright, 2009, "Recovery before redemption: A theory of delays in sovereign debt renegotiations," Working Paper, 1-63.

Chatterjee, S., and B. Eyigungor, 2015, "A seniority arrangement for sovereign debt," American Economic Review, 105, 3740-3765.

Corden, W.M., 1988 , "Debt relief and adjustment incentives," IMF Staff Papers, 35, 628-643.

Curiel, I.J., M. Maschler, and S.H. Tijs, 1987, "Bankruptcy games," Mathematical Methods of Operations Research, 31, 143-159.

D'Erasmo, P., 2011, "Government reputation and debt repayment in emerging economies," Working Paper, 1-49.

Eisenberg, L., and T.H. Noe, 2001, "Systemic risk in financial systems," Management Science, 47, 236-249.

Gillies, D.B., 1959 , "Solutions to general non-zero-sum games," Contributions to the Theory of Games, 4, 47-85. 
Kornai, J., 1979, "Resource-constrained versus demand-constrained systems," Econometrica, 801-819.

Maschler, M., B. Peleg, and L.S. Shapley, 1979, "Geometric properties of the kernel, nucleolus, and related solution concepts," Mathematics of Operations Research, 4, 303338.

Neumann, J. von, and O. Morgenstern, 1944, Theory of games and economic behavior, Princeton University Press, Princeton, New Jersey.

O'Neill, B., 1982, "A problem of rights arbitration from the Talmud," Mathematical Social Sciences, 2, 345-371.

Owen, G., 1974, "A note on the nucleolus," International Journal of Game Theory, 3, 101-103.

Schmeidler, D., 1969, "The nucleolus of a characteristic function game," SIAM Journal on Applied Mathematics, 17, 1163-1170.

Shapley, L.S., 1953, "A value for $n$-person games," in H.W. Kuhn and A.W. Tucker (eds.), Contributions to the Theory of Games, Vol. 2, Annals of Mathematics Studies, 28, Princeton University Press, Princeton, New Jersey, pp. 307-317.

Shapley, L.S., 1971, "Cores of convex games," International Journal of Game Theory, 1, $11-26$.

Sturzenegger, F., and J. Zettelmeyer, 2007, Debt defaults and lessons from a decade of crises, MIT Press, Cambridge, MA.

Thomson, W., 2003, "Axiomatic and game-theoretic analysis of bankruptcy and taxation problems: A survey," Mathematical Social Sciences, 45, 249-297.

Thomson, W., 2013, "Game-theoretic analysis of bankruptcy and taxation problems: Recent advances," International Game Theory Review, 15, 1-14.

Thomson, W., 2015, "Axiomatic and game-theoretic analysis of bankruptcy and taxation problems: An update," Mathematical Social Sciences, 74, 41-59.

Yokote, K., Y. Funaki, and Y. Kamijo, 2014, "Relationship between the Shapley value and other solutions," Working Paper, 1-32.

Yue, V.Z., 2015, "Sovereign default and debt renegotiation," Journal of International Economics, 80, 176-187. 